\title{
The Three Hundred project: the stellar and gas profiles
}

\section{Qingyang Li,${ }^{1 \star}$ Weiguang Cui ${ }^{\oplus},{ }^{2 \star}$ Xiaohu Yang, ${ }^{1,3 \star}$ Elena Rasia ${ }^{\odot},{ }^{4}$ Romeel Dave ${ }^{\oplus}, 2,5$ Marco De Petris, ${ }^{6,7}$ Alexander Knebe ${ }^{\oplus},{ }^{8,9,10}$ John A. Peacock ${ }^{\odot},{ }^{2}$ Frazer Pearce ${ }^{11}$ and Gustavo Yepes ${ }^{8,9}$}

${ }^{1}$ Department of Astronomy, School of Physics and Astronomy, Shanghai Jiao Tong University, Shanghai 200240, China

${ }^{2}$ Royal Observatory, Institute for Astronomy, University of Edinburgh, Edinburgh EH9 3HJ, UK

${ }^{3}$ Shanghai Key Laboratory for Particle Physics and Cosmology, Tsung-Dao Lee Institute, Shanghai Jiao Tong University, Shanghai 200240, China

${ }^{4}$ INAF - Osservatorio Astronomico di Trieste, via Tiepolo 11, I-34143 Trieste, Italy

${ }^{5}$ University of the Western Cape, Bellville, Cape Town 7535, South Africa

${ }^{6}$ Dipartimento di Fisica, Sapienza Università di Roma, P.le Aldo Moro 5, I-00185 Rome, Italy

${ }^{7}$ INFN - Sezione di Roma, P.le Aldo Moro 2, I-00185 Rome, Italy

${ }^{8}$ Departamento de Física Teórica, Módulo 8, Facultad de Ciencias, Universidad Autónoma de Madrid, E-28049 Madrid, Spain

${ }^{9}$ Centro de Investigación Avanzada en Física Fundamental (CIAFF), Universidad Autónoma de Madrid, E-28049 Madrid, Spain

${ }^{10}$ International Centre for Radio Astronomy Research, The University of Western Australia, 35 Stirling Highway, Crawley, Western Australia 6009, Australia

${ }^{11}$ School of Physics, Astronomy, University of Nottingham, Nottingham NG7 2RD, UK

\begin{abstract}
Using the catalogues of galaxy clusters from The Three Hundred project, modelled with both hydrodynamic simulations (GADGET-X and GADGET-MUSIC), and semi-analytical models (SAMs), we study the scatter and self-similarity of the profiles and distributions of the baryonic components of the clusters: the stellar and gas mass, metallicity, the stellar age, gas temperature, and the (specific) star formation rate. Through comparisons with observational results, we find that the shape and the scatter of the gas density profiles matches well the observed trends including the reduced scatter at large radii which is a signature of self-similarity suggested in previous studies. One of our simulated sets, GADGET-X, reproduces well the shape of the observed temperature profile, while GADGET-MUSIC has a higher and flatter profile in the cluster centre and a lower and steeper profile at large radii. The gas metallicity profiles from both simulation sets, despite following the observed trend, have a relatively lower normalization. The cumulative stellar density profiles from SAMs are in better agreement with the observed result than both hydrodynamic simulations which show relatively higher profiles. The scatter in these physical profiles, especially in the cluster centre region, shows a dependence on the cluster dynamical state and on the cool-core/non-cool-core dichotomy. The stellar age, metallicity, and (s)SFR show very large scatter, which are then presented in 2D maps. We also do not find any clear radial dependence of these properties. However, the brightest central galaxies have distinguishable features compared to the properties of the satellite galaxies.
\end{abstract}

Key words: galaxies: clusters: general-galaxies: clusters: intracluster medium-galaxies: general-galaxies: haloes.

\section{INTRODUCTION}

Galaxy clusters are the largest gravitationally bound objects in the universe, containing numerous galaxies, intracluster medium (ICM), and dark matter. Although the baryonic matter only occupies a small fraction (about the cosmic baryonic fraction $\Omega_{\mathrm{b}} / \Omega_{\mathrm{m}}$ ) of the total cluster mass, observing galaxies and the ICM at different

^E-mail: qingyli@sjtu.edu.cn (QL); wcui@ roe.ac.uk (WC); xyang@sjtu.edu.cn (XY) wavelengths, such as optical, X-ray, radio, allows us to measure several cluster properties and to depict a full picture of the cluster. Moreover, the physical property distributions of gas and stars reflect the effect of different physical processes and the formation of the clusters. Therefore, it is essential to understand their distributions and connections, as well as their dependence on the cluster properties/formation history.

It is well known that the density profile of dark matter haloes is self-similar and can be well described by the NFW (Navarro, Frenk \& White 1997) or Einasto (Merritt et al. 2006) fitting 
formulae. Recent developments in hydrodynamic simulations with baryon models indicate that baryons also play a role in shaping the distribution of dark matter (see Cui \& Zhang 2017, for a review). Using the hydro-simulated clusters from The Three Hundred project $^{1}$ (Cui et al. 2018, hereafter C18), Mostoghiu et al. (2019, hereafter M19) showed that the self-similarity of the total cluster density profile, which starts from $z=2.5$, seems to be independent of baryon models (see also Le Brun et al. 2018, for a similar result but with DM-only simulations). However, the total density profile shows dependence on the halo formation time/cluster dynamical state. An open question is whether the gas/stars and their physical property profiles, such as temperature, metallicity, also follow a similar trend and these profiles are model dependent or not.

Clusters have been studied through multiwavelength observations, such as X-ray and optical. X-ray telescopes can detect the high-energy photons scattered by the hot electrons in the ICM via bremsstrahlung emission. These observations provide an insight into the distribution of hot gas (e.g. Böhringer \& Werner 2010). With better X-ray telescopes such as XMM-Newton, Chandra, and Suzaku, the gas properties are investigated with much greater detail (e.g. Majerowicz, Neumann \& Reiprich 2002; Vikhlinin et al. 2005; Sato et al. 2007). Similar to the total density profile, it has been suggested that the hot gas beyond the cooling core region in massive clusters also shows a self-similar evolution in the mean profile up to $z \sim 1.9$ (e.g. Vikhlinin et al. 2006; McDonald et al. 2017). The gas density profile has been well studied in the outskirts from both numerical and observational studies (Roncarelli et al. 2006; Vikhlinin et al. 2006; Lemze et al. 2008). These works found that the outer radii profile can be simply fitted by a power law while in the innermost regions the gas density profile is more cored than the dark matter density profile, even though the trend of the core depends on the cluster dynamical status. Indeed, the cool-core (CC) clusters which have significantly lower temperature gas in the centre and short cooling times show different central gas densities compared with non-cool-core (NCC) clusters.

Also the temperature and the metallicity profiles generally show self-similar profiles at large radii, which can be fitted by a universal fitting function (Mohr, Mathiesen \& Evrard 1999; Vikhlinin et al. 2005; Baldi et al. 2012; Biffi et al. 2018b; Ghirardini et al. 2019). The gas temperature profile slowly increases from the outer regions towards the cluster centre (e.g. Vikhlinin et al. 2005; Pratt et al. 2007; Rasmussen \& Ponman 2007; Reiprich et al. 2009). In the cluster central region, the temperature of $\mathrm{CC}$ and NCC clusters show distinct trends: the CC cluster drops quickly, while the NCC cluster becomes flat (e.g. Sanderson, Ponman \& O'Sullivan 2006; Finoguenov et al. 2007; Dunn \& Fabian 2008). Similarly, the metallicity profile peaked in the centre, decreases with radius, and becomes flat beyond around $\sim 0.3 \times R_{500}{ }^{2}$ (see e.g. Thölken et al. 2016; Ezer et al. 2017; Urban et al. 2017; Vogelsberger et al. 2018; Lovisari \& Reiprich 2019). However, this general trend is more pronounced in CC clusters, which have a significant peak, with respect to NCC objects, which might have a relatively flat profile (e.g. Baldi et al. 2007; Leccardi \& Molendi 2008). Lovisari \& Reiprich (2019) claimed that the metallicity profile is non-uniform by separating the clusters into dynamically relaxed (high concentration and low centroid-shift) and disturbed (low concentration and high centroid-shift) objects. The relaxed systems

\footnotetext{
${ }^{1}$ https://the300-project.org

${ }^{2}$ The subscript 500 or 200 used in this paper refers to enclosed overdensities of 500 or 200 times the critical density of the universe.
}

show a higher metallicity in the centre compared to disturbed systems.

Optical observations have achieved great success in revealing the distribution of galaxies. However, the faint intracluster light (ICL) is still hard to distinguish due to the sensitivity of current telescopes. Normally, stellar population properties are gauged based on modelling the spectral energy distributions of the galaxies. It is also interesting to see whether the stellar properties present a selfsimilar profile or not. Recently, the profile of the star formation rate (SFR) or the specific star formation rate (sSFR) has been investigated in galaxy clusters. Laganá \& Ulmer (2018) (see also Alberts et al. 2016, for a similar result for higher redshift clusters) showed that the SFR seems not to correlate with the projected radius at $0.4<z<0.8$, while the sSFR may also not follow a growing trend with radius as suggested by Brodwin et al. (2013), for example.

Therefore, following M19, we detail the modelled and observed physical profiles and also focus on the difference between models and observations in this paper. We investigate the profiles of stellar properties (stellar mass, age, metallicity) and also study the thermo- and chemodynamical properties of the ICM. We use the multimodelled clusters from both hydrodynamic simulations and semi-analytical models (SAMs) at $z=0$ of The Three Hundred project (Cui et al. 2018; Wang et al. 2018; Arthur et al. 2019; Mostoghiu et al. 2019; Ansarifard et al. 2020; Haggar et al. 2020). A few comparisons are carried out with observations in the X-ray band and optical, using SDSS data.

This paper is organized as follows. In Section 2, we concisely introduce the adopted hydrodynamic simulations and SAMs, as well as the cluster data set. In Section 3, we present the clusters selected from the SDSS 7 catalogue. In Section 4, we present the clusters physical profiles and contrast with observed data. Finally, we summarize our conclusions in Section 5.

\section{THE HYDRODYNAMIC SIMULATIONS AND SEMI-ANALYTICAL MODELS}

Hydrodynamic simulations and SAMs are described in C18 (see also Knebe et al. 2018, for the SAM catalogues), we refer interested readers to those papers for more information. Here, we only briefly summarize some basic details. The 324 regions are centred on galaxy clusters which are initially selected from the MultiDark simulation (Klypin et al. 2016) ${ }^{3}$ - the dark-matter-only MDPL2 with the cosmological parameters from the Planck mission (Planck Collaboration XIII 2016). MDPL2 is a periodic cube of comoving size equal to 1.48 , Gpc containing $3840^{3}$ dark matter particles. The selected 324 galaxy clusters are the most massive objects identified at $z=0$ in the parent simulation. Each re-simulated region has an approximate radius of $\sim 22$, Mpc at $z=0$ which includes the highresolution particles. The outer layer with multiple levels of mass refinement has been generated using the parallel GINNUNGAGAP ${ }^{4}$ code. The hydrodynamic simulations are run with these initial conditions, while the SAMs galaxies of each re-simulation region are cut out from the MultiDark-Galaxies catalogue (Knebe et al. 2018).

We only use the data sets from two simulation codes - GADGETX (Murante et al. 2010; Rasia et al. 2015) and GADGET-MUSIC (Sembolini et al. 2013). Both simulation codes are based on the

\footnotetext{
${ }^{3}$ The MultiDark simulations are publicly available at https://www.cosmos im.org data base.

${ }^{4}$ https://github.com/ginnungagapgroup/ginnungagap
} 
gravity solver of the GADGET3 Tree-PM code (an updated version of the GADGET2 code; Springel 2005) with smoothed particle hydrodynamics (SPH) to follow the evolution of the gas component. GADGET-MUSIC uses the classic entropy-conserving SPH formulation with a 40 neighbour spline kernel, while GADGET-X includes an improved SPH scheme (Beck et al. 2016) with artificial thermal diffusion, time-dependent artificial viscosity, high-order Wendland C4 interpolating kernel and wake-up scheme. GADGET-X is also different from GADGET-MUSIC for the treatment of the baryonic components. Stellar evolution and metal enrichment in GADGET-X (see Tornatore et al. 2007, for the original formulation) consider mass-dependent lifetimes of stars (Padovani \& Matteucci 1993), the production and evolution of 15 different elements coming from SNIa, SNII, and AGB stars with metallicity-dependent radiative cooling (Wiersma, Schaye \& Smith 2009). Although both simulations adopt the stellar feedback model from Springel \& Hernquist (2003), GADGET-MUSIC uses a higher wind velocity $\left(400 \mathrm{~km} \mathrm{~s}^{-1}\right)$ than GADGET-X $\left(350 \mathrm{~km} \mathrm{~s}^{-1}\right)$ for the kinetic stellar feedback. In addition, it also included another mode of thermal feedback - the evaporation of cold clouds due to SN feedback. While GADGET-X models the black hole $(\mathrm{BH})$ growth and implements active galactic nuclei (AGNs) feedback (Steinborn et al. 2015) unlike GADGETMUSIC. We note here that GADGET-MUSIC uses the Salpeter initial mass function (IMF; Salpeter 1955) while GADGET-X applies the Chabrier IMF (Chabrier 2003).

The aforementioned MDPL2 dark-matter-only simulation has been populated with galaxies (Knebe et al. 2018) by three distinct SAMs, i.e. GALACTICUS (Benson 2012), SAG (Cora et al. 2018), and SAGE (Croton et al. 2016). The same 324 regions (using the same radius cut) have also been extracted from the SAMs' haloes and galaxy catalogue that covers the entire simulation volume of the parent MDPL2 simulation. This data set constitutes the counterpart sample from the hydrodynamic catalogue, to which it can be directly compared. All SAMs adopt the Chabrier IMF (Chabrier 2003).

The haloes in each re-simulation region are identified by the Amiga Halo Finder, AHF(Knollmann \& Knebe 2009) using an overdensity threshold of 200 times the critical density of the Universe. In our analysis, we only use the mass-complete clusters. Further, we also recalculate $M_{500}$ and $R_{500}$ for each of the selected clusters using the method presented in Cui, Borgani \& Murante (2014b). Additionally, we recalculate both $R_{500}$ and $R_{200}$ for the MDPL2 haloes used in SAM and corresponding to the hydrodynamical simulated sample (see $\mathrm{C} 18$ for the matching procedure). The SAM galaxies within the corresponding radii are used for comparisons. Finally, to account for our limited mass resolution we select only objects with stellar mass above $5 \times 10^{10} \mathrm{M}_{\odot}$ in both modelled and observed samples.

\section{THE SDSS GALAXY CLUSTERS}

We compared with observations of galaxy clusters from the SDSS 7 catalogue. The SDSS 7 catalogue is taken from Shi et al. (2018) which is based on the Yang et al. (2012) catalogue. This group catalogue is constructed using the adaptive halo-based group finder and halo masses $-M_{200}$ - are assigned to each group using the ranking of either their total characteristic luminosity or total characteristic stellar mass. It uses the same cosmological parameters as the hydrodynamic simulations and SAMs. To consistently make comparisons, we first apply the same halo mass threshold $M_{200}>$ $9.47 \times 10^{14} \mathrm{M}_{\odot}$ to the SDSS group catalogue, as the mass-complete simulated sample. In this way, we select out 100 galaxy clusters including 2905 galaxies from the SDSS 7 catalogue. However, 394 galaxies from the Yang et al. (2018) catalogue do not belong to the SDSS catalogue, so they are excluded. In addition, another 22 galaxies are removed from the catalogue because they are not classified as galaxies in Comparat et al. (2017). We furthermore select galaxies with stellar mass $M_{\star}>5 \times 10^{10} \mathrm{M}_{\odot}$ which is consistent with the simulated catalogue. Eight additional clusters which do not have any galaxies above this mass limit are removed. This results in 1142 galaxies. As the simulation and SAM clusters use the maximum density peak as the centre of the cluster, we consider the brightest/most massive central galaxy (BCG) of the SDSS clusters as the group centre. Finally, the 92 galaxy clusters have 906 satellite galaxies within the projected $r_{200}$. We note here that the mass-weighted mean redshift of these galaxies is $z \approx$ 0.15 .

The stellar population properties of the SDSS galaxies - age, metallicity, stellar mass, and the star formation history - are given by Comparat et al. (2017), who performed full spectral fitting on individual spectra making use of three different high spectral resolution stellar population models: STELIB (Le Borgne et al. 2003), MILES (Sánchez-Blázquez et al. 2006; Beifiori et al. 2011; Falcón-Barroso et al. 2011), and ELODIE (Prugniel et al. 2007). They provided the galaxy properties from different choices of stellar IMF and input stellar libraries, from which we choose the MILES stellar libraries with the Chabrier IMF (Chabrier 2003). We refer to Comparat et al. (2017) for the details of different stellar libraries and IMFs. The SFR and sSFR of these galaxies are taken from Brinchmann et al. (2004).

Even with this stellar mass cut, the galaxies in clusters at high redshift are still incomplete due to the limiting magnitude of current telescopes, i.e. galaxies of the same mass detected at a lower redshift may not be observed at a higher redshift. We use the following method to complete the galaxy members above a certain mass cut. The basic idea is that if a galaxy was observed in a Local Group, it can also be observed in a high-redshift group in which the same mass galaxy is beyond the observational limit. We first correct the galaxy's redshift with $K$ - and $E$-corrections. We further define $z_{\max }$ - the maximum redshift - at which a galaxy with a given stellar mass can be observed by the telescope. In this way, every galaxy has its own $z_{\max }$. Each member galaxy of a selected cluster is referred as the original galaxy. We select all the clusters of which redshifts are lower than an original galaxy's $z_{\max }$. Then, we consider that all these clusters should include this galaxy. However, because there is only one galaxy among these clusters, we conclude that the probability that this original galaxy can be observed in these selected clusters is $1 / N_{\mathrm{c}}$, where $N_{\mathrm{c}}$ is the total number of the selected clusters with $z<z_{\max }$. Meanwhile, all the clusters whose redshifts are lower than this galaxy $z_{\max }$ should contain this galaxy with the same probability, $1 / N_{c}$. Only satellite galaxies are considered here. These quantities are added to the clusters with redshift larger than the galaxy $z_{\max }$ at the same radius of the original galaxy. With this method applied, an additional $\sim 1990$ galaxies are included in our SDSS sample, which results in 2896 satellite galaxies in total. But we only apply this method to the complete catalogue sample in calculating the stellar number density and mass density. This possibility is directly taken account as the satellite galaxy number for the calculation of satellite number density. While it is multiplied with the original galaxy stellar mass for the calculation of stellar mass density. For the other stellar properties, such as age and metallicity, we only use these member galaxies and do not take this incompleteness correction into account. 


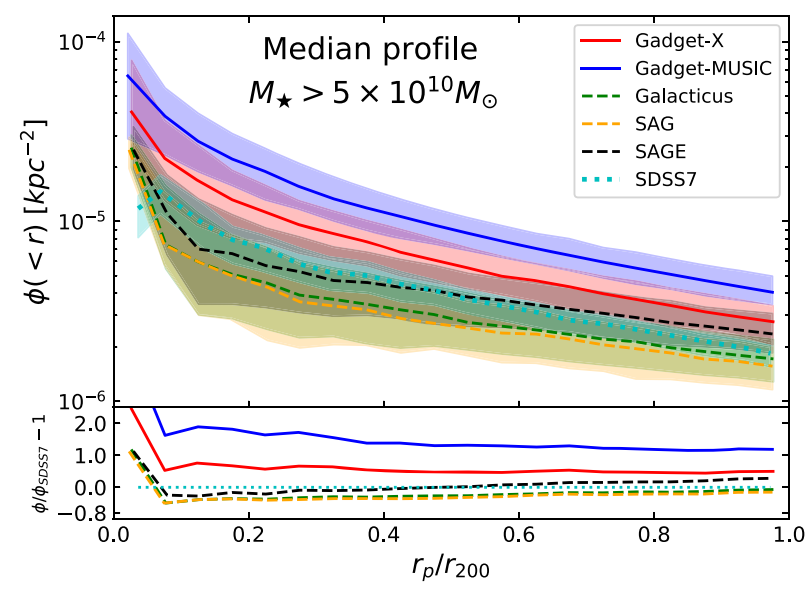

Figure 1. Cumulative galaxy number density as a function of the normalized radius. The same galaxy stellar mass limit $\left(5 \times 10^{10} \mathrm{M}_{\odot}\right)$ is applied to all modelled and observed galaxies. As indicated in the legend, different colour and line styles represent the median profile from different models and the observed SDSS result. The shaded regions are the 16th and 84th percentiles of all cluster profiles. The bottom panel shows the residuals compared with the SDSS7 data.

\section{RESULTS}

We present here the scatter and the universality of the physical profiles of galaxy clusters. Physical profiles, separated into stellar and gas components, are presented in Sections 4.1 and 4.2, respectively. For the stellar physical profiles, we focus on stellar density, age, and metallicity, which are all derived only from the satellite galaxies. ${ }^{5}$ For the gaseous physical profiles, we investigate the gas density, temperature, metallicity, SFR, and sSFR properties, which are based only on the gas content from the two hydrodynamical runs. It is worth noting that the radius for all the stellar and gas profiles is normalized to $r_{200}$ and $R_{500}$, respectively, in order to compare with the observational results. Throughout the paper, $r$ indicates the projected radius (only member galaxies within $R_{200}$ are included in the projection), while $R$ is the distance in 3D. We only select the $x-y$ plane to project these simulated clusters. These profiles are generally shown via medians with the error bar indicating the 16 th and 84th percentiles in each radius bin. The solar metallicity is taken from Asplund et al. (2009) with the value $Z_{\odot}=0.0134$.

\subsection{Stellar physical profiles}

Due to the fact that all the stellar properties investigated here are derived from satellite galaxies, we first check our data consistency by presenting the cumulative galaxy number density $-\phi-$ in the selected galaxy clusters in Fig. 1. Here, $\phi$ is defined as $N(<r) / \pi r^{2}$ where $N(<r)$ is the total galaxy number within $r$. We also show the residuals using each data set in comparison with the SDSS measurements. Apparently, SAMs are in better agreement with observation than the two hydro-simulations. GADGET-MUSIC, which does not have AGN feedback, has the highest galaxy number which is $\sim 2$ times higher than the SDSS observation. GADGET-X, even with AGN feedback, still has about 50 per cent more galaxies than the SDSS result. This decreasing trend of the number density profiles indicates that the satellite galaxy number density drops from inner

${ }^{5}$ Both the BCG and the ICL are not taken into account, unless specified.

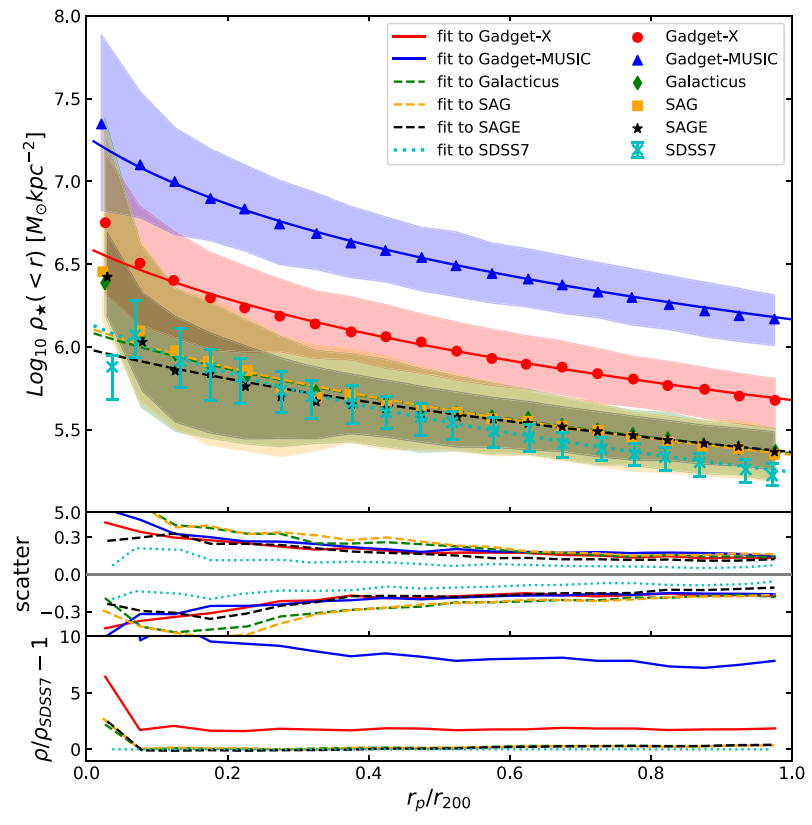

Figure 2. Cumulative stellar mass density profile as a function of the normalized radius. Different models and observed median profiles are shown in different colour symbols, while shaded regions (for models) and error bars (for the SDSS observation) present 16th and 84th percentiles of all cluster profiles. The middle panel highlights these scatters. The bottom panel shows the fitting residuals compared with the SDSS result. Fitting results of median data with the formula from Łokas \& Mamon (2001) are presented in Table 1.

to outer radii. We note here that this galaxy mass cut may bias our results towards high-mass galaxies, such as more red or old galaxies which may not be well modelled in the two hydro-simulations (more details can be found in Section 4.3). The drop of the innermost data point from SDSS clusters could be caused by a projection effect with miss-identification of galaxies (this data point is very close to the edge of the BCG) or by a bias which is coming from the offset between the BCG and the number density peak of the galaxy clusters.

\subsubsection{Stellar mass profile}

The stellar mass density profile indicates how the stars/galaxies are distributed in the cluster environments. As the galaxy cluster is at the final stage of structure formation, this stellar mass density profile could potentially be a powerful tool to constrain galaxy formation and evolution. However, it is not easy to measure the observed stellar component in the cluster accurately. This is because the ICL is very faint, normally below the telescope detection limit, and it can contribute a significant amount of stellar mass (see e.g. Cui et al. 2014a), especially at the cluster centre region. Therefore, we only use the satellite galaxy stellar mass from both models and observation here to estimate this stellar mass density profile.

The cumulative stellar mass density profile, which uses the galaxy mass above the mass cut, is presented in Fig. 2. The fitting function with the form of the NFW profile (Navarro et al. 1997) is obtained from Łokas \& Mamon (2001):

$M(s)=M_{200} g(c)\left(\ln (1+c s)-\frac{c s}{1+c s}\right)$,

where 
$s=\frac{r}{r_{200}}$,
$c=\frac{r_{200}}{r_{\mathrm{s}}}$,

$g(c)=\frac{1}{\ln (1+c)-\frac{c}{1+c}}$.

Finally, the density profile can be expressed as

$\rho=\frac{M(s)}{V}$,

where volume $V$ equals $\pi r^{2}$ in projection and $4 / 3 \pi R^{3}$ in real space. We note here that this fitting function was originally used for dark matter density profiles. It seems that the stellar density profile follows a similar profile to dark matter. So we use this function as the fitting function, only replacing the total mass, $M_{200}$, by the total satellite stellar mass, $M_{*, 200}$. We exclude the innermost data point from these fits. The scatter and fitting residuals are, respectively, presented in the middle and bottom panel of Fig. 2. The residuals are calculated by comparing with the fitted SDSS result. In agreement with Fig. 1, the stellar mass density profiles are also basically in alignment with the SDSS result, except GADGET-MUSIC and GADGET-X. The almost constant shift of the GADGET-MUSIC and GADGET-X profiles with respect to the result from SDSS indicates that (1) the AGN feedback has a homogeneous effect on the galaxy mass that does not depend on the distance to the cluster centre; (2) the density profile from GADGET-X, even with AGN feedback, is still about 2 times higher than the profile from SDSS; (3) GADGETMUSIC, which does not include AGN feedback and has a weaker SN feedback (as implied by its higher satellite galaxy stellar mass function in Cui et al. 2018) compared to GADGET-X, presents a much higher (about 4 times) stellar density profile. The error bars are at a level of $\sim 0.5$ dex depending on the models. This indicates that, like the halo density profile, the stellar density profile of the galaxy cluster is almost universal. We study the origin of this scatter by separating our clusters into relaxed and un-relaxed clusters in Appendix A1, which only has a weak impact for the outer radii. The fitting parameters are listed in Table 1 .

\subsubsection{Stellar age distribution}

For modelled galaxies, age is the mass-weighted age of all stars inside the $z=0$ galaxy. We find that the mean and mass-weighted ages are very similar as the stellar particles have similar masses. The age of an observed galaxy is derived from model fitting of the galaxy spectrum (see details in Comparat et al. 2017). For SDSS galaxies with low S/N spectra, SED model fits will tend to overestimate the age. This is because the stellar model fit is independent of cosmology. Therefore, we exclude about 120 galaxies which have their age older than the Universe (13.79 Gyr) in this analysis. The satellite galaxies' age distributions is shown in Fig. 3. GALACTICUS is excluded from this plot due to the lack of galaxy age. Histograms of the galaxy ages are shown in the right-hand sub-panels. The age of BCGs from hydrodynamic simulations is defined as the massweighted star particle age within $0.015 \times R_{200}$. We investigated varying this to the larger radius of $0.05 \times R_{200}$ and did not find any significant changes in the BCG ages.

First, there is a very large scatter in the age distributions of both the SDSS and modelled galaxies. This indicates a significant mix of young and old galaxies at all radii in the cluster environment. Although we exclude galaxies older than the Universe, there is a noticeable fraction of very old galaxies. This is due to the model fitting. Secondly, the satellite galaxies in both hydro-simulations are primarily dominated by old galaxies with ages of $\sim 11 \mathrm{Gyr}$, which are not apparent in either the SAMs or SDSS. Thirdly, unlike the SAMs and the SDSS, the BCGs from both hydro-simulations are much younger than their satellite galaxies. The reason could be that star formation is not fully quenched by AGN activity in the cluster centre, which is especially clear in the GADGET-MUSIC run.

\subsubsection{Stellar metallicity distribution}

Stellar metal enrichment is mainly determined by stellar nucleosynthesis, which is correlated with the chosen IMF, initial metallicity and age. Therefore, the galaxy stellar metallicity is directly related to its age.

The galaxy stellar metallicity distributions with respect to the solar metallicity for the two hydrodynamic simulations, SAMs and the SDSS 7 galaxies are presented in Fig. 4. The metallicity of the BCGs in the hydrodynamic simulations is defined as the massweighted star particles' metallicity within $0.015 R_{200}$. Again, we do not find any significant change on the BCG metallicities when this limiting radius is taken to be 10 times larger. The striking point is that the disagreement between the models and observation is quite large. The galaxy metallicity from models is generally less than the solar metallicity, while the majority of SDSS galaxies have metallicity $\sim 1.5-2 \times Z_{\odot}$. The sharp cut-off in the SDSS metallicity map is due to the limitation of the SED fitting model. When we compare the galaxy metallicity distribution with their age distribution, we find that the modelled galaxies with a greater age tend to have a lower metallicity. This can be understood as the metallicity is dominated by younger stars, which have a higher initial metallicity because of their later formation. However, SDSS galaxies tend to have both older age and higher metallicity, which does not fit into this picture. This could be due to the intrinsic simple stellar population (SSP) fitting, which may provide different views on the galaxy age and metallicity. In particular, this SSP fitting is strongly model dependent (Comparat et al. 2017). The ELODIE-type models give a distribution of metallicities stretching towards sub-solar values while the MILES-based models used in this work remain more concentrated at solar metallicities. The STELIB library grants a smaller coverage in metallicity, hence model results are confined between half-solar and twice-solar in chemical composition. The range in ages found using STELIB-based models is larger and extends to younger ages with respect to the other two models. If we put aside the uncertainty in the SSP fitting and assume that the disagreement between the models and observation is real, one possible solution for models is to form more young stars. However, this will also bring down the age profile. Therefore, this dilemma should perhaps instead be solved via other methods such as a higher metal production in $\mathrm{SN}$ feedback.

\subsection{Gas profiles}

It is widely known that gas properties, such as density, temperature, pressure, and entropy, show self-similar profiles (see among other papers Vikhlinin et al. 2006; Cavagnolo et al. 2009; Arnaud et al. 2010; Baldi et al. 2012; Planelles et al. 2017; Biffi, Mernier \& Medvedev 2018a; Ghirardini et al. 2019). Moreover, it has been proposed that gas metallicity is homogeneously distributed near the outer radii of clusters (see e.g. Biffi et al. 2017; Mantz et al. 2017; Vogelsberger et al. 2018). However, gas physical profiles (especially 
Table 1. The fitting function (equation 5, based on Łokas \& Mamon 2001) and parameters for Fig. 2. $M_{*, 200}$ is set to the total stellar mass of satellite galaxies within $r_{200}$. Here, we use the median value of $r_{200}$ and $M_{*, 200}$ for these clusters (shown in the second and third rows) in the fitting function and fit the free parameter $c$ with the median density profiles of each model.

\begin{tabular}{lcccccc}
\hline Parameters & GADGET-X & GADGET-MUSIC & GALACTICUS & SAG & SAGE & SDSS \\
\hline$r_{200}\left(10^{3} \mathrm{kpc}\right)$ & 2.246 & 2.251 & 2.251 & 2.251 & 2.251 & 2.294 \\
$M_{*, 200}\left(10^{12} \mathrm{M}_{\odot}\right)$ & 7.558 & 23.319 & 3.615 & 3.546 & 3.681 & 2.893 \\
Free parameter $c$ & 3.486 & 4.896 & 2.368 & 2.539 & 1.810 & 3.356 \\
\hline
\end{tabular}
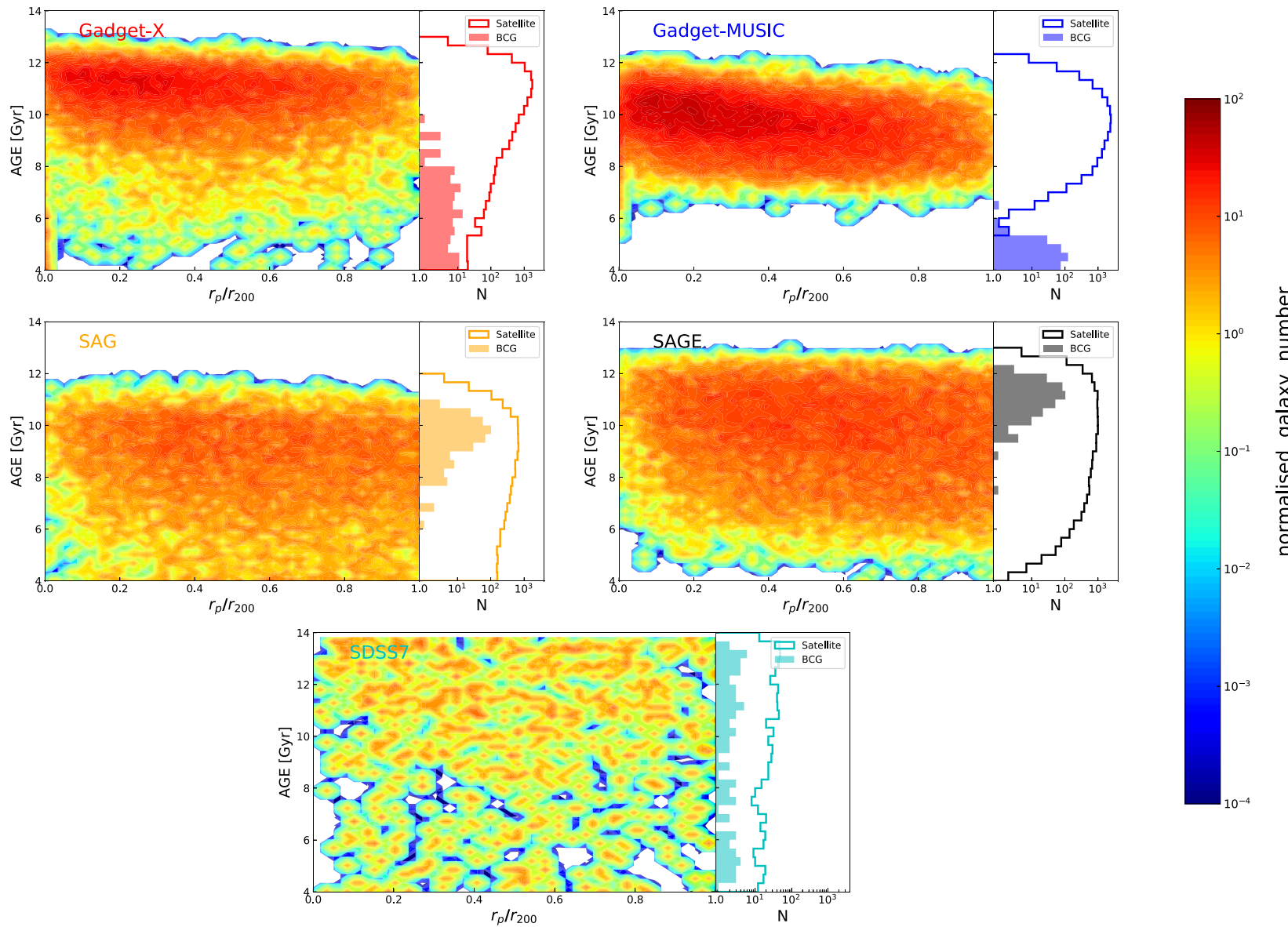

Figure 3. The distribution of satellite galaxy age as a function of radius inside the clusters. No BCGs are included in the left-hand panels. The right-hand sub-panels show the age histograms for the BCGs (filled) and satellite galaxies (solid). The colour bar shows the normalized galaxy number density with respect to the total cluster number. A Gaussian filter with standard deviation of 0.5 pixels is applied to smooth these maps.

in cluster centres) seem to depend on the cluster dynamical state (e.g. Lovisari \& Reiprich 2019) or on the CC/NCC classification (e.g. Baldi et al. 2007; Leccardi \& Molendi 2008).

We present gas profiles from the two hydrodynamic simulations and also compare with observational results in this section. We note here that the gas profiles are calculated by summing over all the gas particles within the cluster. Because SAMs only provide gas properties within galaxies, which cannot be compared with observed results from X-ray telescopes, we do not include SAMs in this section. To compare with X-ray observational results of the smoothly distributed hot gas within the cluster, we select gas particles in the simulation with a temperature, $T>0.3 \mathrm{keV}$, and gas density, $\rho<0.1 \mathrm{~cm}^{-3}$ i.e. lower than the star-forming threshold. We calculate the gas temperature profile using the spectroscopic- like formula from Mazzotta et al. (2004):

$T_{\mathrm{sl}}=\frac{\Sigma_{i} m_{i} \rho_{i} T_{i}^{1 / 4}}{\Sigma_{i} m_{i} \rho_{i} T_{i}^{-3 / 4}}$,

where $m_{i}$ is gas mass, $\rho_{i}$ is gas density, and $T_{i}$ is gas temperature of each considered gas particle. The distribution of the estimated gas temperatures in the clusters is shown in Table 3.

For the metallicity, we consider the simplified emission-weighted formula:

$Z_{\mathrm{EW}}=\frac{\Sigma_{i} m_{i} \rho_{i} \sqrt{T_{i}} Z_{i}}{\Sigma_{i} m_{i} \rho_{i} \sqrt{T_{i}}}$.

The metallicity profile uses emission-weighted gas metallicity which is normalized to solar metallicity with respect to Asplund 

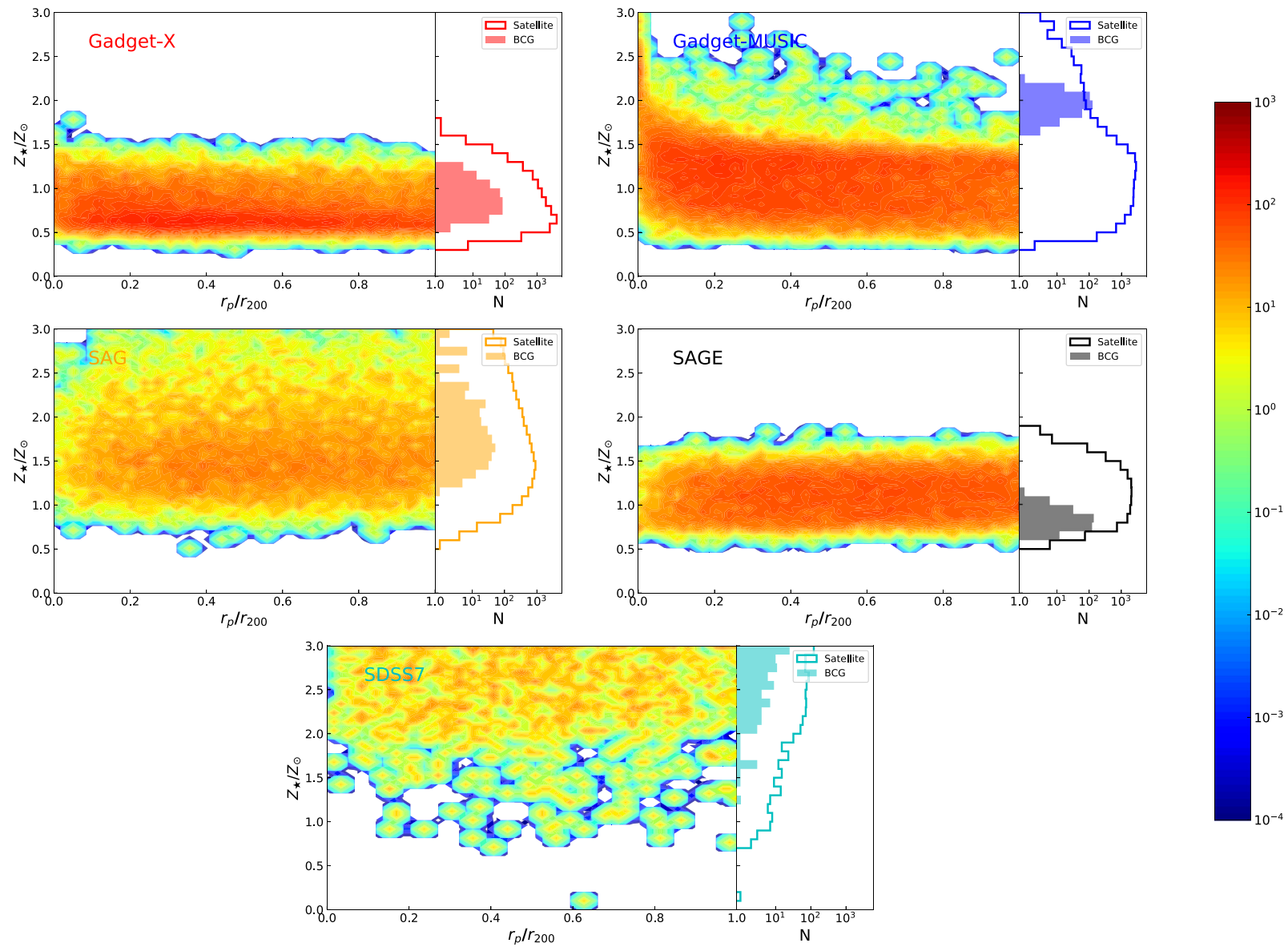

Figure 4. Similar to the age distribution in Fig. 3 but for the stellar metallicity distribution of the satellite galaxies inside the clusters. The BCG distributions are only included in the right-hand panels as filled steps.

Table 2. The double $\beta$-model fitting function (based on Mohr et al. 1999) and fitting parameters for Fig. 5.

\begin{tabular}{lccccc}
\hline Fitting parameters & $\rho_{1}\left(\mathrm{M}_{\odot} \mathrm{kpc}^{-3}\right)$ & $R_{1}\left(R_{500}\right)$ & $\rho_{2}\left(\mathrm{M}_{\odot} \mathrm{kpc}^{-3}\right)$ & $R_{2}\left(R_{500}\right)$ & $\beta$ \\
\hline GADGET-X & $8.027 \times 10^{4}$ & 0.240 & $2.304 \times 10^{5}$ & 0.059 & 0.770 \\
GADGET-MUSIC & $2.773 \times 10^{5}$ & 0.045 & $8.372 \times 10^{4}$ & 0.210 & 0.751 \\
Fitting function & & $\rho(R)=\sum_{i=1}^{2} \rho_{i}\left[1+\left(\frac{R}{R_{i}}\right)^{2}\right]^{-3 \beta / 2}$ & \\
\hline
\end{tabular}

Table 3. The minimum, median, and maximum spectroscopiclike temperatures of the clusters.

\begin{tabular}{lccr}
\hline Simulation & & $\begin{array}{c}T_{500, \mathrm{sl}}(\mathrm{keV}) \\
\text { Median }\end{array}$ & Max \\
\hline GADGET-X & 2.68 & 5.67 & 12.56 \\
GADGET-MUSIC & 2.46 & 4.92 & 9.94 \\
\hline
\end{tabular}

et al. (2009) $\left(Z_{\odot}=0.0134\right)$. To have a consistent comparison with our simulation data, only observational data on clusters with $z<$ 0.1 and $M_{500}>4.0 \times 10^{14} \mathrm{M}_{\odot}$ from observations are considered in this subsection. Finally, we normalize the profile with respect to $R_{500}$ as done in observations.

\subsubsection{Gas density profile}

Previous studies, using dark-matter-only simulations (Le Brun et al. 2018), full physics hydrodynamic simulations (M19) and observations at cluster scales (e.g. McDonald et al. 2017), have revealed that both the total mass profile and the gas density profile show self-similar behaviours out to redshift $\sim 2$ in the outer region. We revisit this feature here by comparing with observed profiles to detail any differences and to understand the physics behind this behaviour.

The gas density profile is presented in Fig. 5, where we compare two hydrodynamic simulation results with the gas density profile from various observed data. Three clusters selected from Vikhlinin et al. (2006) with the criteria listed in the last paragraph of Section 4.2 are shown by dashed lines: A478, A1795, and 


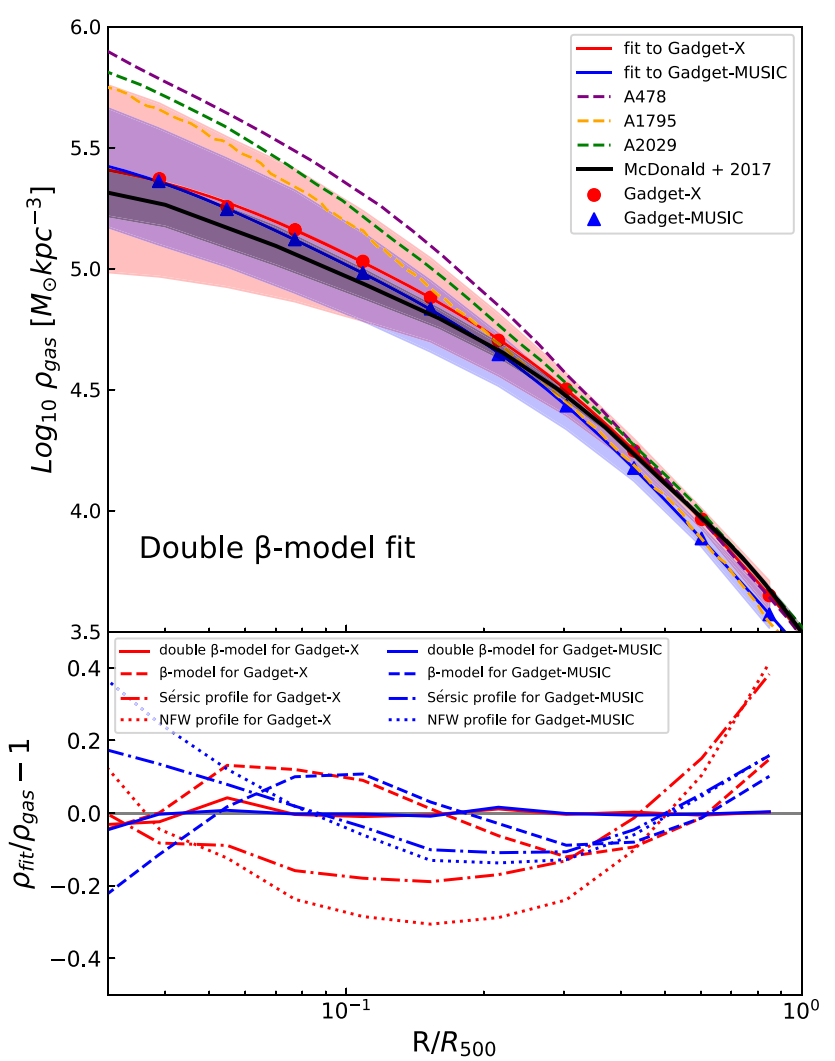

Figure 5. Radial gas mass density profile for two hydrodynamic simulations. Red circles and blue triangles, respectively, represent the median profiles from GADGET-X and the GADGET-MUSIC runs with solid lines for their best fit with a double $\beta$-model. Shaded areas for two hydrodynamic simulations show the 16th and 84th percentiles of all cluster profiles. Purple, orange, and green dashed lines show gas density profiles of three galaxy clusters: A478, A1795, and A2029, respectively, which are taken from Vikhlinin et al. (2006). The black solid line shows the median gas density profile with $1 \sigma$ uncertainty for galaxy clusters at $0.0<z<0.1$ from McDonald et al. (2017). Bottom panels are the residuals between different the fitting function and the median data points for GADGET-X (red) and GADGET-MUSIC (blue). Solid, dashed, dash-dotted, and dotted lines, respectively, show different fitting functions: double $\beta$-model, $\beta$-model, Sérsic profile, and NFW profile. The fitting results are shown in Table 2.

A2029; the median gas density profile at $0.0<z<0.1$ is taken from McDonald et al. (2017) which uses 27 clusters with masses spanning $4 \times 10^{14} \mathrm{M}_{\odot}<\mathrm{M}_{500}<1.2 \times 10^{15} \mathrm{M}_{\odot}$. This sample is $\mathrm{X}$-ray flux-limited and constrained in redshift, as originally selected in Vikhlinin et al. (2009). It has a similar number of CC, moderate $\mathrm{CC}$, and NCC clusters.

The top panel also shows the results of fitting the double $\beta$-model (Mohr et al. 1999). The residuals between the median and fits with four different models are presented in the bottom panel. The four models are, respectively, the double $\beta$-model (Mohr et al. 1999), the $\beta$-model (Cavaliere \& Fusco-Femiano 1976), the Sérsic profile (Sérsic 1963) and the NFW profile (Navarro et al. 1997) shown with solid, dashed, dash-dotted, and dotted lines for GADGET-X (red) and GADGET-MUSIC (blue). The Sérsic and NFW fitting functions used here are mainly for a simple comparison to the distribution that is typically assumed for the stellar and the DM components.

The gas density profile is highly peaked towards the centre, which is in agreement with many observational results (see e.g. Pointecouteau et al. 2004). And this is also the reason why the

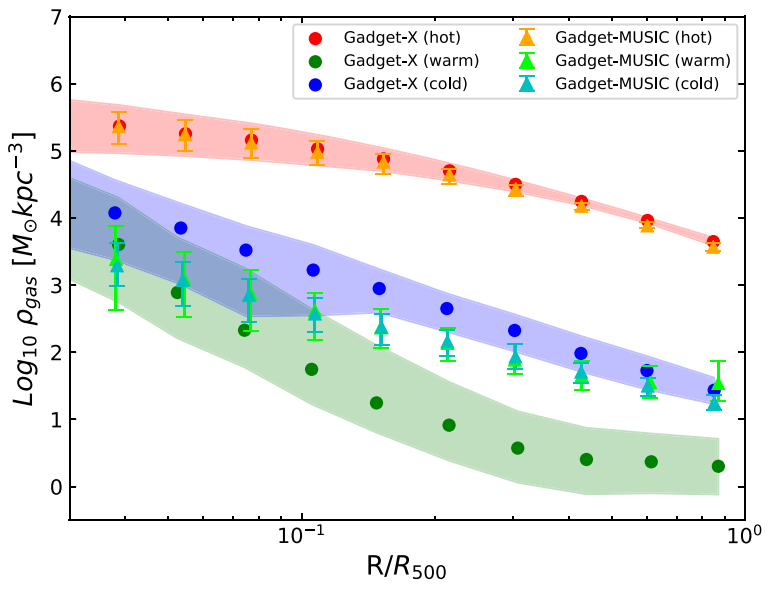

Figure 6. Gas density profiles for gas at different temperatures (in the case of three temperature ranges, see the text). Circles and triangles represent, respectively, GADGET-X and GADGET-MUSIC. Red (orange), green (lime), and blue (cyan) circles show hot, warm, and cold gas median density profiles from GADGET-X (GADGET-MUSIC). Shaded regions (for GADGET-X) and error bars (for GADGET-MUSIC) present the 16th and 84th percentiles of all cluster profiles.

best-fitting function is the double $\beta$-model. At outer cluster radii, the gas density profile between the hydro-simulations (GADGET-X and GADGET-MUSIC) and observations (three clusters A478, A1795, and A2029 from Vikhlinin et al. 2006 and McDonald et al. 2017) shows a consistent trend, but larger discrepancies are present in the central region $\left(r \lesssim 0.1 \times R_{500}\right)$. Compared with the two hydrosimulations, the three individual clusters and McDonald et al. (2017), respectively, give a higher and lower density. However, error bars also significantly increase in the cluster centre, indicating gas density changes between individual clusters. This inner scatter can be reduced by separating clusters into cool core and non-cool core or into dynamically relaxed and un-relaxed (see more details in Appendix A1). This means that the density profile is sensitive to both the implemented physical models and the detailed halo formation history. Overall, the agreements between two hydro-simulations and between the simulations and observational results are relatively good. This confirms the self-similarity of the gas profiles and further indicates that baryon models play a weak role in shaping the gas density profiles, especially in the outer regions, where gas follows the distribution of dark matter. Therefore, it is also not surprising to see that gas density profiles with much less scatter display a similar trend to the stellar density profile, even though they are much steeper in the outer regions.

The total gas density profiles of the hot component of the clusters produced by GADGET-X and GADGET-MUSIC are very similar. We, then, investigate whether gas at different temperatures shows similar trends between the two simulations. We first separate the gas into hot $\left(>10^{7} \mathrm{~K}\right)$, warm $\left(10^{7}-10^{5} \mathrm{~K}\right)$ and cold $\left(<10^{5} \mathrm{~K}\right)$ phases (see e.g. Cui et al. 2019, for a similar definition). As shown in Fig. 6, the gas mass is dominated by hot gas in clusters and our two simulations also share a similar profile for hot gas, even though GADGET-MUSIC is slightly lower at the outer radii compared to GADGET-X in agreement with Fig. 5. The warm and cold gas profiles from GADGET-MUSIC are very similar and also close to the cold gas profile from GADGET$\mathrm{X}$, all of which are about two orders of magnitude lower than the hot gas profile. The warm gas profile from GADGET-X is lower than the others, which may be because GADGET-X uses a much more 


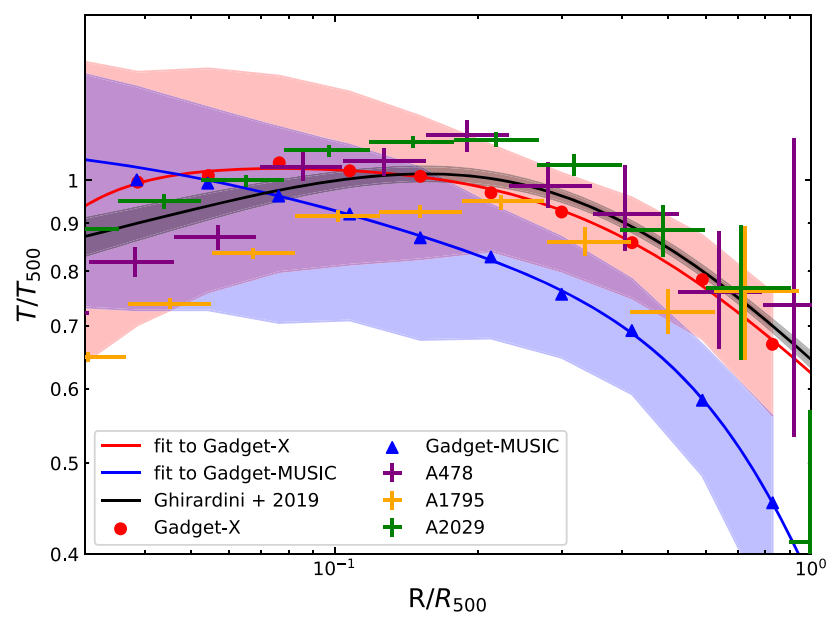

Figure 7. Radial spectroscopic-like gas temperature profile. Red circles and blue triangles, respectively, represent the median profile from GADGET-X and GADGET-MUSIC where the shaded areas show the 16th and 84th percentiles of all cluster profiles. We note here that an additional normalization factor is applied to the two simulations; please refer to the text for details. Red and blue solid lines show fits of the formula from Ghirardini et al. (2019). Purple, orange and green crosses, respectively, represent cluster A478, A1795, and A2029 temperature profiles obtained from Vikhlinin et al. (2006). The black solid line with fitting percentile error shown as a shaded region is from Ghirardini et al. (2019). The fitting results are listed in Table 4.

efficient cooling rate (metal cooling) than GADGET-MUSIC (which has metal independent cooling). In the very inner cluster region all the different profiles converge towards $\rho_{\text {gas }} \approx 10^{5.5} \mathrm{M}_{\odot} \mathrm{kpc}^{-3}$.

\subsubsection{Gas temperature profile}

It is well known that the cluster gas temperature shows a tight scaling relation with its mass (see $\mathrm{C} 18$, and references therein). Observations also suggested that the gas temperature profile shows similarity after rescaling with cluster mass (e.g. Vikhlinin et al. 2005; Pratt et al. 2007; Rasmussen \& Ponman 2007; Baldi et al. 2012; Ghirardini et al. 2019). Ghirardini et al. (2019), for example, rescaled the temperature profiles derived for the X-ray Cluster Outskirts Project (X-COP, Eckert et al. 2017) clusters as

$T_{500, G+19}=8.85 \mathrm{keV}\left(\frac{M_{500}}{10^{15} h_{70}^{-1} \mathrm{M}_{\odot}}\right)^{2 / 3} E(z)^{2 / 3} \frac{\mu}{0.6}$,

where $E(z)$ is defined as $E^{2}(z)=\Omega_{\mathrm{m}}(1+z)^{3}+\Omega_{\Lambda}$ and $\mu$ is the mean molecular weight per gas particle, which Ghirardini et al. (2019) assumed to be equal to 0.6125 (Anders \& Grevesse 1989). Consistent with Ghirardini et al. (2019), we adopt the same $\mu$ for calculating the gas temperature $T_{\mathrm{sl}}$ from the simulations and use $T_{500, G+19}$ for the normalization. However, we find that $T_{500, G+19}$ from equation (8) with the $M_{500}$ from our simulation is a little higher than $T_{500, \mathrm{sl}}$. That could be due to the fact that $M_{500}$ in $T_{500, G+19}$ is based on the hydrostatic equilibrium assumption, which tends to give a lower value. As shown in C18, there is a slight (about 14 per cent) off-set in the $M_{500}-T_{500}$ relation between the hydro-simulations and observation. We make a detailed investigation of the difference between $T_{500, \mathrm{sl}}$ and $T_{500, G+19}$ and find a similar deviation, which is presented in Appendix B. Therefore, we apply this correction fraction to our simulation result in Fig. 7.

In Fig. 7, the gas temperature profiles are compared, with A478, A1975, and A2029 from Vikhlinin et al. (2006) shown by data

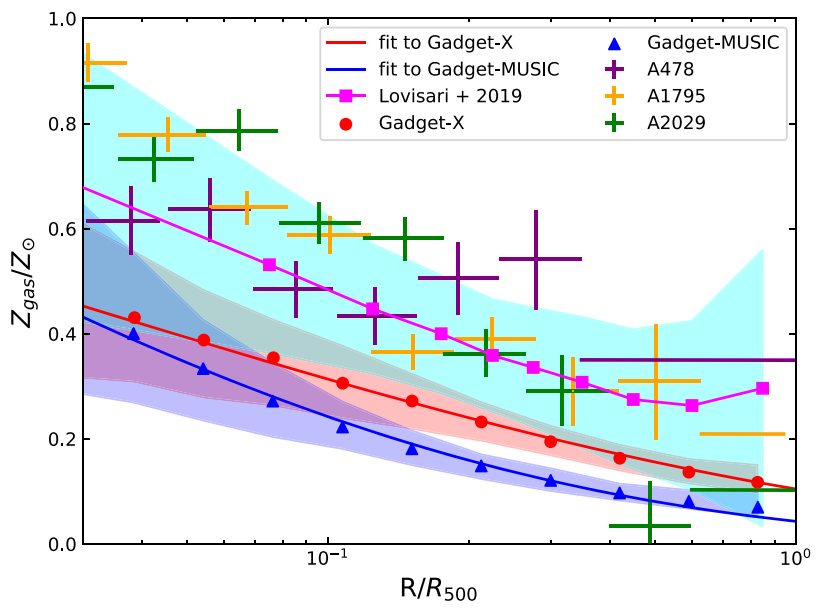

Figure 8. Radial emission-weighted gas metallicity profile. The red circles and blue triangles, respectively, represent GADGET-X and GADGET-MUSIC where solid lines and shaded areas show the fits with a Sérsic profile and the 16th and 84th percentiles of all cluster profiles, respectively. The purple, orange, and green crosses show the gas metallicity profile of the galaxy clusters: A478, A1795, and A2029, respectively, taken from Vikhlinin et al. (2005). The magenta rectangles and cyan region show the median abundance profile and scatter of measurements given by Lovisari \& Reiprich (2019).

points with error bars and the fitting result from Ghirardini et al. (2019) shown by a solid black line plus a grey-shaded region. These three clusters from Vikhlinin et al. (2006) adopt the same normalization with $T_{500, G+19}$. The profile by Ghirardini et al. (2019) is based on a total number of 12 clusters which are originally selected from the first Planck Sunyaev-Zel'dovich (SZ) catalogue (Planck Collaboration XXIX 2014) with an SZ signal limitation and low redshift $(0.04<z<0.1)$. This sample has mass range $3 \times 10^{14} \mathrm{M}_{\odot}<\mathrm{M}_{500}<9 \times 10^{14} \mathrm{M}_{\odot}$ with $4 \mathrm{CC}$ and $8 \mathrm{NCC}$ based on the central entropy value measured by Cavagnolo et al. (2009).

Observed temperature profiles show a slightly increasing trend from the cluster centre outwards to $r \sim 0.2 \times R_{500}$ and decrease from 20 per cent of $R_{500}$ to the outer regions. The gas temperature profile from GADGET-X is in good agreement with observations, while GADGET-MUSIC has a much higher and flatter profile in the cluster centre compared to observations. Its profile in the outer regions is also lower and slightly steeper than the observed results. This could be caused by the normalization. Unlike the gas density profile, the gas temperature profile is strongly affected by the baryon models. Overall, there are large scatters in these two hydro-simulations, especially in the cluster centre region. We further test whether this scatter is caused by cluster dynamical state and CC/NCC classification in Appendix A1 and find that the scatter in the cluster centre regions can be significantly reduced by separating the clusters into $\mathrm{CC} / \mathrm{NCC}$.

\subsubsection{Gas metallicity profile}

The metal enrichment of the ICM involves numerous astrophysical processes, such as stellar nucleosynthesis and supernova explosions. The resulting metals will enrich the surrounding ICM thanks to multiscale mixing processes, such as galactic winds, AGN feedback, ram-pressure stripping and mergers. Both observation (e.g. Leccardi \& Molendi 2008; Werner et al. 2013; McDonald et al. 2016; Mantz et al. 2017) and simulations (e.g. Biffi et al. 
Table 4. The fitting function from Ghirardini et al. (2019) and parameters for Fig. 7.

\begin{tabular}{lcccccc}
\hline Fitting parameters & $T_{0}\left(T_{500}\right)$ & $T_{\min }\left(T_{500}\right)$ & $r_{\text {cool }}\left(R_{500}\right)$ & $a_{\text {cool }}$ & $r_{t}\left(R_{500}\right)$ & $c$ \\
\hline GADGET-X & 1.046 & 0.212 & 0.016 & 3.170 & 0.393 & 0.515 \\
GADGET-MUSIC & 0.759 & 1.120 & 0.099 & 1.242 & 1.141 & 2.574 \\
Fitting function & \multicolumn{5}{c}{$\frac{T(x)}{T_{500}}=T_{0} \frac{\frac{T_{\min }}{T_{0}}+\left(\frac{x}{r_{\text {cool }}}\right)^{a_{\text {cool }}}}{1+\left(\frac{x}{r_{\text {cool }}}\right)^{a_{\text {cool }}}} \frac{1}{\left(1+\left(\frac{x}{r_{t}}\right)^{2}\right)^{\frac{c}{2}}}$} \\
\end{tabular}

Table 5. The fitting function and parameters for Fig. 8.

\begin{tabular}{lcc}
\hline Fitting parameters & GADGET-X & GADGET-MUSIC \\
\hline$Z_{0}\left(\mathrm{Z}_{\odot}\right)$ & 0.602 & 0.669 \\
$R_{0}\left(R_{500}\right)$ & 0.001 & 0.002 \\
$b$ & 0.419 & 0.863 \\
& & $Z_{0} \mathrm{e}^{-b\left[\left(\frac{R}{R_{0}}\right)^{\frac{1}{4}}-1\right]}$ \\
\hline
\end{tabular}

2017; Biffi et al. 2018a; Vogelsberger et al. 2018) have suggested a homogeneous distribution of metals in space and time. The uniform metal distributions in the outskirts of nearby clusters indicates an early enrichment of the ICM, most of which takes place before cluster formation.

Fig. 8 shows the emission-weighted gas metallicity profile as a function of radius normalized to $R_{500}$. The metallicity profile is compared with the stacked profile by Lovisari \& Reiprich (2019), who study galaxy cluster metallicity profiles using a sample of 207 nearby galaxy groups and clusters observed with XMM-Newton. The stacked profile is estimated with a Monte Carlo method based on performing 10000 realizations of the profiles by randomly varying the observational data points of the metallicity profile. Besides this stacked data set we also compare to three individual cluster results from Vikhlinin et al. (2006).

In general, the radial gas metallicity profile is centrally peaked and gradually decreases from the centre to $0.2-0.3 R_{500}$, where it flattens and stays almost constant out to large radii. Both simulations and observations follow the same trend. However, the simulations are slightly lower (much lower metallicity in GADGET-MUSIC than GADGET-X) than the observed data points at the outer radii. As shown in Rasia et al. (2008), the metallicity derived from XMMNewton spectra of simulated mock observations was generally in good agreement with the emission-weighted metallicity. So this difference must arise for other reasons. For GADGET-X, we especially compare to Biffi et al. (2018a) which showed a good match to the observational results and find that this lower metallicity is mainly caused by a lower star formation and inefficient kinetic SN feedback in the GADGET-X run, therefore less metal is produced. The lower star formation is introduced by several parameter changes compared to Biffi et al. (2018a), driven mostly by the choice of a much larger gravitational softening length. Furthermore, as indicated in Vogelsberger et al. (2018), this could be a resolution issue, which is also consistent with our previous findings. Because of the modest resolution of these simulations, the star formation starts later in time leading to a reduced amount of metals in both stars and diffuse gas. Similarly to the observed profile (see also Elkholy, Bautz \& Canizares 2015; Mernier et al. 2016), gas metallicity becomes basically flat at $r>R / R_{500}$. The error bars of simulated profiles for both GADGET-X and GADGET-MUSIC are very narrow at outer radii: this indicates that the variation of gas metallicity between clusters is very small and that the detailed cluster formation history has a limited effect on the metal enrichment of the recent accreted ICM. The AGN feedback from GADGET-X seems to have a non-negligible effect by boosting gas metallicity at larger radii compared to GADGET-MUSIC as found in Rasia et al. (2015) and Biffi et al. (2018a). We further fit the metallicity profile from the two simulations with the Sérsic profile, which provides a very good match to the simulation data. The fitting results are listed in Table 5 .

\subsection{SFR and SSFR distributions}

The SFR serves as a connection between gas and stars. It also determines galaxy colour and connects to many galaxy properties such as shape and age. Many studies have revealed that the number fraction of red, quiescent (blue star-forming) galaxies decreases (increases) with increasing halo-centric radius in the cluster (see e.g. Weinmann et al. 2006; Bai et al. 2009). Using 69 local clusters, Chung et al. (2011) claimed that the sSFR increases with projected radius after applying an SFR cut $\left(L_{\mathrm{IR}}>4.7 \times 10^{10} \mathrm{~L}_{\odot}\right)$, which is consistent with the study of $\mathrm{H} \alpha$ star-forming galaxies by Lewis et al. (2002). However, Laganá \& Ulmer (2018) studied 17 galaxy clusters at intermediate-to-high redshifts $(0.4<z<0.9)$ and found that the mean SFR of both SF galaxies and quenched galaxies does not change with cluster-centric radius. Similar results for clusters at higher redshifts $(1.0<z<1.75)$ were also found by Alberts et al. (2016). It is interesting to see whether the modelled galaxies are in agreement with these findings at lower redshifts.

The galaxy SFR is directly provided by SAMs, while the galaxy SFR from hydro-simulations is calculated by summing up the instantaneous SFR from gas that lies within twice its stellar halfmass radius. We also compute the galaxy SFR via its mass change from the previous snapshot divided by the time difference between the two snapshots which is about $300 \mathrm{Myr}$. Both methods give very similar SFR values. Therefore, we use the galaxy SFR from the first method here. The SFRs of SDSS galaxies are estimated through their $\mathrm{H} \alpha$ luminosities (Brinchmann et al. 2004). Rather than firstly separating the star-forming galaxies from quenched galaxies with some arbitrary values, we divide the data into 60 bins in both radius and SFR or sSFR. Any galaxy with SFR value below $0.001 \mathrm{M}_{\odot} \mathrm{yr}^{-1}$ is set at $0.001 \mathrm{M}_{\odot} \mathrm{yr}^{-1}$; meanwhile, its sSFR is set at $10^{-15} \mathrm{yr}^{-1}$. Each pixel value is normalized by the total cluster number. Finally, a Gaussian filter with standard deviation 0.5 pixels is used to smooth both the SFR and the sSFR 2D images.

In Figs 9 and 10, we show, respectively, the distribution of galaxy SFR and sSFR for satellite galaxies. Each panel shows one model with the colour-bar indicating a normalized galaxy number density. Solid, dashed and dotted black lines, respectively, represent the 16th, 50th, and 84th percentiles of pixel value from the SDSS results. These lines are repeated in each panel for reference. We also show histograms for the BCG (only presented in the histogram with filled 

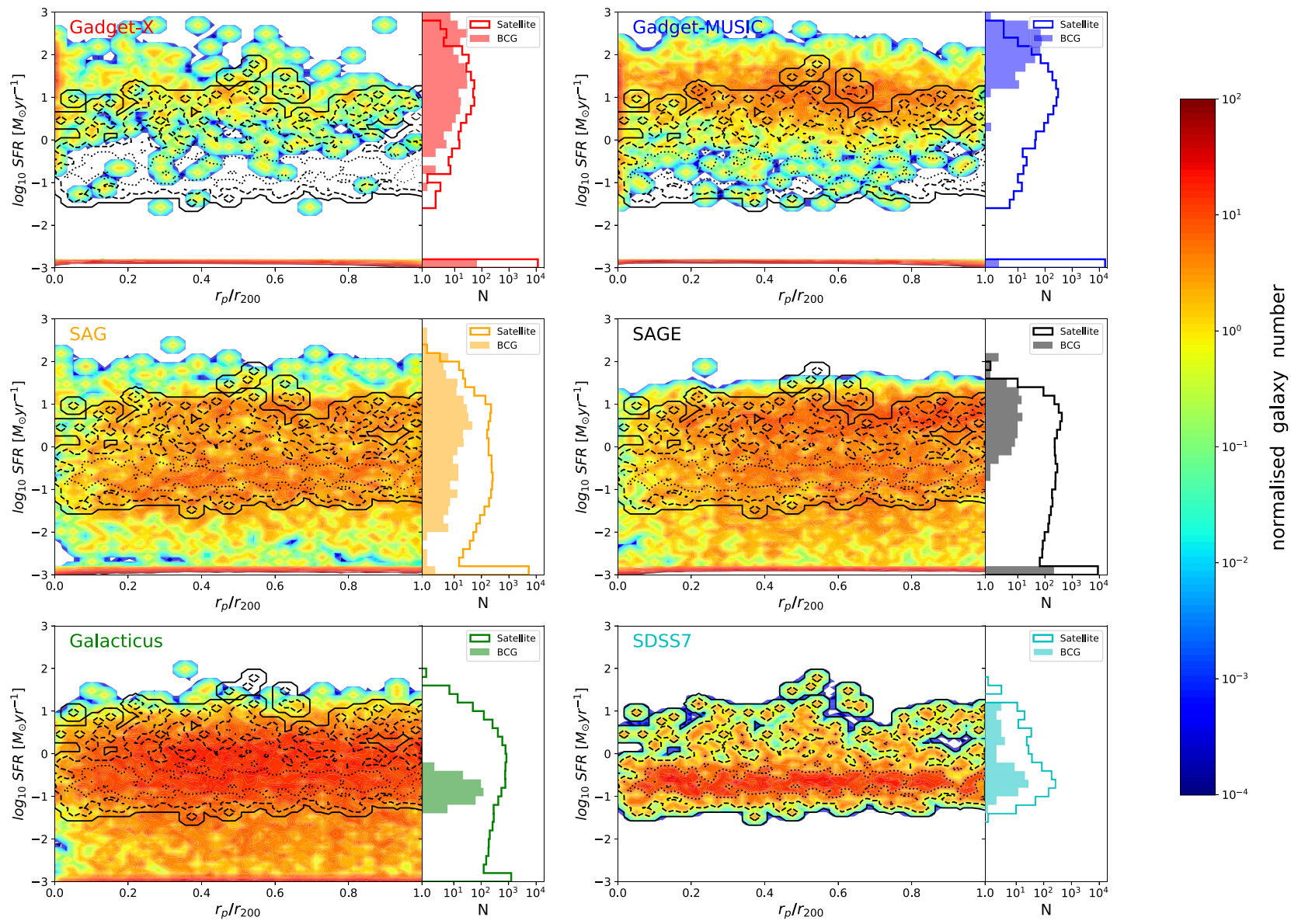

Figure 9. The distribution of satellite galaxy SFR with radius inside clusters. No BCGs are included in the left-hand panels. The colour bar shows the normalized galaxy number density with respect to total cluster number. The SFR histograms for BCGs (filled) and satellite galaxies (solid) are shown in the right-hand sub-panels. Solid, dashed, and dotted black lines, respectively, represent the 16th, 50th, and 84th percentiles of the pixel value from SDSS result, which is repeated in all panels for comparison. A Gaussian kernel is used to smooth the pixels. All galaxies with SFR less than $0.001 \mathrm{M}_{\odot} \mathrm{yr}^{-1}$ are put in the lowest SFR bin.

steps) and satellite galaxy SFRs in the right-hand sub-panels. The SFR of the BCGs from the hydrodynamic simulations is defined as the total SFR from all gas particles within $0.015 \times R_{200}$, while the sSFR of the BCGs is calculated by SFR/ $M_{\text {star }}$ within $0.015 \times R_{200}$. We find only a few clusters from GADGET-MUSIC do not contain gas particles within $0.015 \times R_{200}$. We again test that extending this limiting radius to $0.05 \times R_{200}$ and find that only GADGET-MUSIC has its BCG's SFR dropping slightly.

For galaxy SFR, it is clear that the most galaxies have an SFR less than $1 \mathrm{M}_{\odot} \mathrm{yr}^{-1}$ for all models and SDSS result. However, the detailed SFR distributions differ between these models and SDSS galaxies: there is no observed galaxy with SFR < $0.01 \mathrm{M}_{\odot} \mathrm{yr}^{-1}$, although this could reflect observational limitations. All of the modelled galaxies have a significant fraction with SFR $<0.001 \mathrm{M}_{\odot} \mathrm{yr}^{-1}$; for the modelled galaxies with SFR $>$ $0.001 \mathrm{M}_{\odot} \mathrm{yr}^{-1}$, SAMs have much smoothed distribution filling the whole plot and tend to have a better agreement to the SDSS distribution, while the two hydro-simulations present more starforming galaxies and fewer galaxies between $1 \mathrm{M}_{\odot} \mathrm{yr}^{-1}>\mathrm{SFR}>$ $0.001 \mathrm{M}_{\odot} \mathrm{yr}^{-1}$. Overall, the radial distribution of the galaxy SFR is more or less constant, which seems to be in agreement with the high-redshift results (Alberts et al. 2016; Laganá \& Ulmer 2018) rather than the low-redshift results (Weinmann et al. 2006; Bai et al.
2009; Chung et al. 2011). If the star-forming galaxies are selected out from SDSS with SFR $>1 \mathrm{M}_{\odot} \mathrm{yr}^{-1}$, we can see their number fractions decrease towards the cluster centre. In agreement with the SFR distribution, the SSFR distribution also does not show much evolution with radius. Again, the contours from the two hydrodynamic simulations seem a little higher than SDSS result, while SAGE and GALACTICUS have fewer galaxies with and a higher sSFR compared to the SDSS result. This indicates that high SFR galaxies in SAGE and GALACTICUS are too massive as compared to SDSS galaxies. From the histogram of BCG SFR, SDSS seems to have a double peak with the majority of BCGs having a quenched SFR $<1 \mathrm{M}_{\odot} \mathrm{yr}^{-1}$. Apart from this the BCGs from GALACTICUS are in good agreement with SDSS, all the other models have their BCG SFR widely spread and the BCGs in the two hydrosimulations tend to have a higher SFR than their star-forming galaxies.

\section{CONCLUSIONS}

We have studied the physical profiles of galaxy clusters using 324 massive clusters from two hydrodynamic simulations and modelled by three SAMs at $z=0$. Observations including massive galaxy groups from the SDSS catalogue and many X-ray data sets are used to compare galaxy and gas properties of our models with 

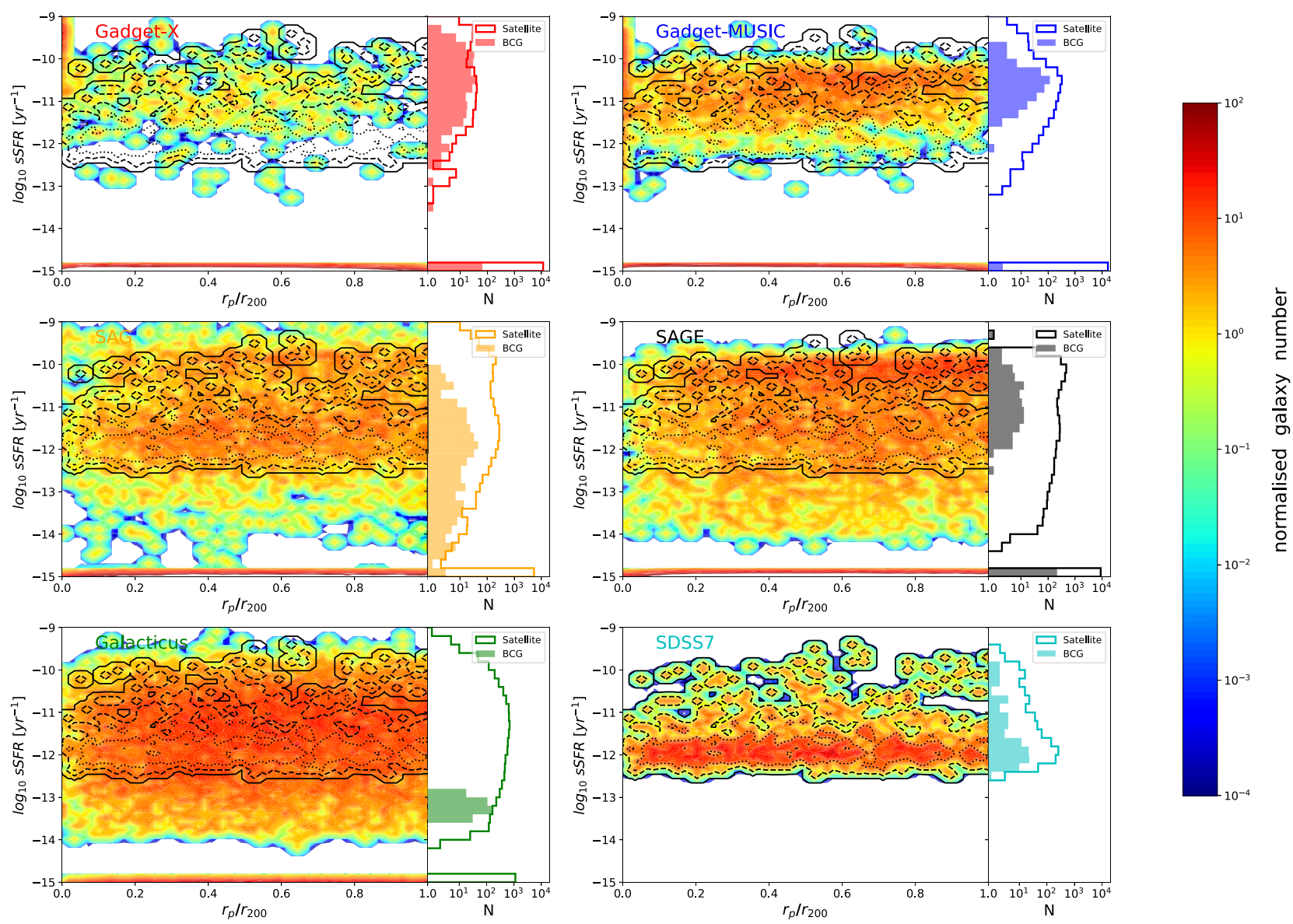

Figure 10. Similar to the SFR distribution in Fig. 9 but for the SSFR distribution of satellite galaxies inside the clusters. The BCG distributions are only included in the right-hand panels as filled steps. All galaxies with sSFR less than $10^{-15} \mathrm{yr}^{-1}$ are put in the lowest sSFR bin.

reality. For stellar properties, we consider galaxy stellar density, galaxy age and metallicity after applying the same mass cut for both modelled and SDSS galaxies. For gas properties, we study the gas density, temperature and metallicity. We also further investigate SFR and sSFR distributions as a function of halo-centric radius. The followings are our main conclusions.

Regarding self-similarity, the gas density profiles from both sets of simulated clusters present the highest level of similarity and are characterized by very small scatter at outer radii. There is a slightly larger scatter in the cluster centre indicating that different physical states of the cluster play a role, such as dynamical state or CC/NCC dichotomy.

The simulated gas metallicity profiles also have a very small (similar) scatter at the cluster outer radii. This scatter is smaller than what is typically observed and could be due to systematic effects in the metallicity measurements. However, the normalization of the simulated metallicity profiles tends to be lower than the observed results.

Both gas temperature profiles and cumulative stellar mass profiles display a relatively large scatter, implying that these quantities are more influenced by the detailed halo formation history. By separating the clusters into relaxed and un-relaxed groups, we further show in Appendix A1 that relaxed clusters tend to have a higher (lower) temperature (stellar density) profile compared to un-relaxed ones. Furthermore, not surprisingly, the CC clusters have a lower temperature profile in the cluster centre than NCC clusters.
Galaxy age, metallicity and (s)SFR do not present any clear radial distributions and have a large scatter at a given radius. Therefore, they are presented in a 2D map for detailed investigation and comparison. They do not show any clear sign of radial dependence.

In general, it is important to compare the simulated cluster results with observations in order to understand whether the baryon models in the simulations impact on the shape and normalization of the profiles. The gas density profiles present the best agreement between different models and observations. This means that the gas density at outer radii $\left(R>0.2 \times R_{500}\right)$ depends only weakly on the baryon models. GADGET-X simulated clusters have a median temperature profile that agrees with observation with a correct shape, while GADGET-MUSIC show a higher and flatter temperature profile in the cluster centre with a steeper and lower temperature profile at large radii compared to observation. The modelled gas metallicity profiles are slightly lower than the observed ones with GADGET-X closer to the observed results at middle and outer radii and GADGET-MUSIC closer in the innermost region. Several reasons might generate this shift including the modest resolution of our sample which could delay star formation and therefore metal production.

For the cumulative stellar mass profile, the three SAMs similarly present a good match to the SDSS result. However, both GADGET$\mathrm{X}$ and GADGET-MUSIC show much higher (about 2 and 8 times, respectively) stellar profiles than the one from SDSS. It seems that we need even stronger feedback to match the SDSS result. 
We also find some discrepancies between models and observation when comparing the 2D distributions of galaxy age, metallicity and (s)SFR: The BCGs from the observed clusters and from the SAMs have a relatively old age, which is not seen in both hydrosimulations; observed galaxies tend to have higher metallicity than the models; both SFR and sSFR show similar distributions between SAM modelled galaxies and the observed clusters for the galaxies with SFR $>0.1 \mathrm{M}_{\odot} \mathrm{yr}^{-1}$. However, the two hydro-simulations show larger deviations from the observations. Besides GALACTICUS, the BCG SFR from all the other models tend to have a significant fraction with values greater than 1 . Finally, it is hard to draw a firm conclusion because, on one hand, the different theoretical models do not present consistent results and on the other hand, there is a large uncertainty in the quantities derived from observations.

There are two remaining questions that are not answered in the previous investigations: (1) What causes the scatter in these physical profiles? (2) What is the redshift evolution of these profiles during the formation of clusters? For the first one, we only partly study the effect of the dynamical state (and CC/NCC separation) of these clusters on the stellar density, gas density, temperature and metallicity profiles in Appendix A1. It is known that the dynamical state correlates with the cluster formation time (see e.g. Mostoghiu et al. 2019) which indeed plays a role in the scatter of galaxy profiles (see Appendix A1 for details). However, to fully understand the physics behind this, we need census and correlation studies with halo properties, which will be detailed in a following work. For the second question, we will trace the halo progenitors and investigate their physical profiles at different redshifts. This is also planned for a further forthcoming paper.

\section{ACKNOWLEDGEMENTS}

The authors thank the referee for his/her valuable suggestions and helps. The authors sincerely thank Giuseppe Murante, Stefeno Borgani, and Klaus Dolag for their works on the GADGET-X development, Johan Comparat and Claudia Maraston for their kind helps and useful discussions on the SDSS firefly catalogue, Veronica Biffi for discussions on gas profiles, Vittorio Ghirardini for his kind helps on the temperature profiles, and Lorenzo Lovisari for his helps on the gas metallicity profile. The authors also thank Yang Lei for the calculation of $K$ - and $E$-correction of SDSS galaxies and Robert Mostoghiu for useful discussions.

This work has made extensive use of the PYTHON packages - IPYTHON with its JUPYTER notebook (Pérez \& Granger 2007), NUMPY (van der Walt, Colbert \& Varoquaux 2011), and SCIPY (Oliphant 2007; Millman \& Aivazis 2011). All the figures in this paper are plotted using the PYTHON MATPLOTLIB package (Hunter 2007). This research has made use of NASA's Astrophysics Data System and the arXiv preprint server.

The CosmoSim data base used in this paper is a service by the Leibniz-Institute for Astrophysics Potsdam (AIP). The MultiDark data base was developed in cooperation with the Spanish MultiDark Consolider Project CSD2009-00064.

The authors gratefully acknowledge the Gauss Centre for Supercomputing e.V. (www.gauss-centre.eu) and the Partnership for Advanced Supercomputing in Europe (PRACE, www.prace-ri.eu) for funding the MultiDark simulation project by providing computing time on the GCS Supercomputer SuperMUC at Leibniz Supercomputing Centre (LRZ, www.lrz.de).

WC and JAP acknowledge supported from the European Research Council under grant number 670193. AK and GY are supported by the Ministerio de Economía y Competitividad and the Fondo Europeo de Desarrollo Regional (MINECO/FEDER, UE) in Spain through grant AYA2015-63810-P and MICIU/FEDER through grant PGC2018-094975-C21. AK further acknowledges funding through the Spanish Red Consolider MultiDark FPA201790566-REDC and thanks Blumfeld for l'etat et moi. XY is supported by the National Science Foundation of China (NSFC, grant nos. 11890692, 11833005, 11621303) and the 111 Project no. B20019.

As requested by 'The Three Hundred' policy, we state the contribution by each author to this paper: QL, WC, and XY formed the core team to do the analysis, produce the plots and write the paper; WC developed and supervised the project; ER. provided critical feedback, contributed to the $\mathrm{CC} / \mathrm{NCC}$ data, and the interpretation of the results; RD, MDP, JAP, and FP provided critical feedback for this work. WC and GY contributed to the simulated data set. AK contributed to the halo catalogue. The author list is in alphabetic order with the exclusion of the first four authors.

\section{REFERENCES}

Alberts S. et al., 2016, ApJ, 825, 72

Anders E., Grevesse N., 1989, Geochim. Cosmochim. Acta, 53, 197

Ansarifard S. et al., 2020, A\&A, 634, A113

Arnaud M., Pratt G. W., Piffaretti R., Böhringer H., Croston J. H., Pointecouteau E., 2010, A\&A, 517, A92

Arthur J. et al., 2019, MNRAS, 484, 3968

Asplund M., Grevesse N., Sauval A. J., Scott P., 2009, ARA\&A, 47, 481

Bai L., Rieke G. H., Rieke M. J., Christlein D., Zabludoff A. I., 2009, ApJ, 693, 1840

Baldi A., Ettori S., Mazzotta P., Tozzi P., Borgani S., 2007, ApJ, 666, 835

Baldi A., Ettori S., Molendi S., Gastaldello F., 2012, A\&A, 545, A41

Beck A. M. et al., 2016, MNRAS, 455, 2110

Beifiori A., Maraston C., Thomas D., Johansson J., 2011, A\&A, 531, A109

Benson A. J., 2012, New Astron., 17, 175

Biffi V. et al., 2017, MNRAS, 468, 531

Biffi V., Mernier F., Medvedev P., 2018a, Space Sci. Rev., 214, 123

Biffi V., Planelles S., Borgani S., Rasia E., Murante G., Fabjan D., Gaspari M., 2018b, MNRAS, 476, 2689

Böhringer H., Werner N., 2010, A\&AR, 18, 127

Brinchmann J., Charlot S., White S. D. M., Tremonti C., Kauffmann G., Heckman T., Brinkmann J., 2004, MNRAS, 351, 1151

Brodwin M. et al., 2013, ApJ, 779, 138

Cavagnolo K. W., Donahue M., Voit G. M., Sun M., 2009, ApJS, 182, 12

Cavaliere A., Fusco-Femiano R., 1976, A\&A, 500, 95

Chabrier G., 2003, PASP, 115, 763

Chung S. M., Eisenhardt P. R., Gonzalez A. H., Stanford S. A., Brodwin M., Stern D., Jarrett T., 2011, ApJ, 743, 34

Comparat J. et al., 2017, preprint (arXiv:1711.06575)

Cora S. A. et al., 2018, MNRAS, 479, 2

Croton D. J. et al., 2016, ApJS, 222, 22

Cui W., Zhang Y., 2017, The Impact of Baryons on the Large-Scale Structure of the Universe. InTech Open Science

Cui W. et al., 2014a, MNRAS, 437, 816

Cui W., Borgani S., Murante G., 2014b, MNRAS, 441, 1769

Cui W., Power C., Borgani S., Knebe A., Lewis G. F., Murante G., Poole G. B., 2017, MNRAS, 464, 2502

Cui W. et al., 2018, MNRAS, 480, 2898

Cui W. et al., 2019, MNRAS, 485, 2367

Dunn R. J. H., Fabian A. C., 2008, MNRAS, 385, 757

Eckert D., Ettori S., Pointecouteau E., Molendi S., Paltani S., Tchernin C., 2017, Astron. Nachr., 338, 293

Elkholy T. Y., Bautz M. W., Canizares C. R., 2015, ApJ, 805, 3

Ezer C., Bulbul E., Nihal Ercan E., Smith R. K., Bautz M. W., Loewenstein M., McDonald M., Miller E. D., 2017, ApJ, 836, 110

Falcón-Barroso J., Sánchez-Blázquez P., Vazdekis A., Ricciardelli E., Cardiel N., Cenarro A. J., Gorgas J., Peletier R. F., 2011, A\&A, 532, A95 
Finoguenov A., Ponman T. J., Osmond J. P. F., Zimer M., 2007, MNRAS, 374,737

Ghirardini V. et al., 2019, A\&A, 621, A41

Haggar R., Gray M. E., Pearce F. R., Knebe A., Cui W., Mostoghiu R., Yepes G., 2020, MNRAS, 492, 6074

Hunter J. D., 2007, Comput. Sci. Eng., 9, 90

Klypin A., Yepes G., Gottlöber S., Prada F., Heß S., 2016, MNRAS, 457, 4340

Knebe A. et al., 2018, MNRAS, 474, 5206

Knollmann S. R., Knebe A., 2009, ApJS, 182, 608

Laganá T. F., Ulmer M. P., 2018, MNRAS, 475, 523

Le Borgne J. F. et al., 2003, A\&A, 402, 433

Le Brun A. M. C., Arnaud M., Pratt G. W., Teyssier R., 2018, MNRAS, 473, L69

Leccardi A., Molendi S., 2008, A\&A, 487, 461

Lemze D., Barkana R., Broadhurst T. J., Rephaeli Y., 2008, MNRAS, 386, 1092

Lewis I. et al., 2002, MNRAS, 334, 673

Łokas E. L., Mamon G. A., 2001, MNRAS, 321, 155

Lovisari L., Reiprich T. H., 2019, MNRAS, 483, 540

McDonald M. et al., 2016, ApJ, 826, 124

McDonald M. et al., 2017, ApJ, 843, 28

Majerowicz S., Neumann D. M., Reiprich T. H., 2002, A\&A, 394, 77

Mantz A. B., Allen S. W., Morris R. G., Simionescu A., Urban O., Werner N., Zhuravleva I., 2017, MNRAS, 472, 2877

Mazzotta P., Rasia E., Moscardini L., Tormen G., 2004, MNRAS, 354, 10

Mernier F., de Plaa J., Pinto C., Kaastra J. S., Kosec P., Zhang Y. Y., Mao J., Werner N., 2016, A\&A, 592, A157

Merritt D., Graham A. W., Moore B., Diemand J., Terzić B., 2006, AJ, 132, 2685

Millman K. J., Aivazis M., 2011, Comput. Sci. Eng., 13, 9

Mohr J. J., Mathiesen B., Evrard A. E., 1999, ApJ, 517, 627

Mostoghiu R., Knebe A., Cui W., Pearce F. R., Yepes G., Power C., Dave R., Arth A., 2019, MNRAS, 483, 3390

Murante G., Monaco P., Giovalli M., Borgani S., Diaferio A., 2010, MNRAS, 405, 1491

Navarro J. F., Frenk C. S., White S. D. M., 1997, ApJ, 490, 493

Oliphant T. E., 2007, Comput. Sci. Eng., 9, 10

Padovani P., Matteucci F., 1993, ApJ, 416, 26

Pérez F., Granger B. E., 2007, Comput. Sci. Eng., 9, 21

Planck Collaboration XXIX, 2014, A\&A, 571, A29

Planck Collaboration XIII, 2016, A\&A, 594, A13

Planelles S. et al., 2017, MNRAS, 467, 3827

Pointecouteau E., Arnaud M., Kaastra J., de Plaa J., 2004, A\&A, 423, 33

Pratt G. W., Böhringer H., Croston J. H., Arnaud M., Borgani S., Finoguenov A., Temple R. F., 2007, A\&A, 461, 71

Prugniel P., Soubiran C., Koleva M., Le Borgne D., 2007, preprint (astro-p $\mathrm{h} / 0703658)$

Rasia E., Mazzotta P., Bourdin H., Borgani S., Tornatore L., Ettori S., Dolag K., Moscardini L., 2008, ApJ, 674, 728

Rasia E. et al., 2015, ApJ, 813, L17

Rasmussen J., Ponmanv T. J., 2007, MNRAS, 380, 1554

Reiprich T. H. et al., 2009, A\&A, 501, 899

Roncarelli M., Ettori S., Dolag K., Moscardini L., Borgani S., Murante G., 2006, MNRAS, 373, 1339

Salpeter E. E., 1955, ApJ, 121, 161

Sánchez-Blázquez P. et al., 2006, MNRAS, 371, 703

Sanderson A. J. R., Ponman T. J., O’Sullivan E., 2006, MNRAS, 372, 1496

Sato K. et al., 2007, PASJ, 59, 299

Sembolini F., Yepes G., De Petris M., Gottlöber S., Lamagna L., Comis B., 2013, MNRAS, 429, 323

Sérsic J. L., 1963, Bol. Asoc. Argentina Astron. La Plata Argentina, 6, 41

Shi F. et al., 2018, ApJ, 861, 137

Springel V., 2005, MNRAS, 364, 1105

Springel V., Hernquist L., 2003, MNRAS, 339, 289

Steinborn L. K., Dolag K., Hirschmann M., Prieto M. A., Remus R.-S., 2015, MNRAS, 448, 1504
Thölken S., Lovisari L., Reiprich T. H., Hasenbusch J., 2016, A\&A, 592, A37

Tornatore L., Borgani S., Dolag K., Matteucci F., 2007, MNRAS, 382, 1050 Urban O., Werner N., Allen S. W., Simionescu A., Mantz A., 2017, MNRAS, 470,4583

van der Walt S., Colbert S. C., Varoquaux G., 2011, Cumput. Sci. Eng., 13, 22

Vikhlinin A. et al., 2009, ApJ, 692, 1033

Vikhlinin A., Markevitch M., Murray S. S., Jones C., Forman W., Van Speybroeck L., 2005, ApJ, 628, 655

Vikhlinin A., Kravtsov A., Forman W., Jones C., Markevitch M., Murray S. S., Van Speybroeck L., 2006, ApJ, 640, 691

Vogelsberger M. et al., 2018, MNRAS, 474, 2073

Wang Y. et al., 2018, ApJ, 868, 130

Weinmann S. M., van den Bosch F. C., Yang X., Mo H. J., 2006, MNRAS, 366,2

Werner N., Urban O., Simionescu A., Allen S. W., 2013, Nature, 502, 656

Wiersma R. P. C., Schaye J., Smith B. D., 2009, MNRAS, 393, 99

Yang X., Mo H. J., van den Bosch F. C., Zhang Y., Han J., 2012, ApJ, 752, 41

Yang X. et al., 2018, ApJ, 860, 30

\section{APPENDIX A: THE EFFECTS OF CLUSTER DYNAMICAL STATES AND CC/NCC DICHOTOMY}

The profiles investigated in this paper such as the gas density profile show a very strong self-similarity, i.e. very small error bars in the profile. While some have relatively large error bars, such as the stellar density and gas temperature profiles. M19 showed that the total density profile depends on the cluster dynamical state, i.e. the relaxed clusters have a higher scale radius $r_{\mathrm{s}}$ than un-relaxed clusters, which essentially links with the halo formation time. Therefore, we naively expect that the cluster dynamical state is also responsible for the large dispersion in the physical profiles. In this section, we separate the clusters into relaxed and un-relaxed (Cui et al. 2017), CC and NCC (Rasia et al. 2015) and try to understand whether cluster dynamical state and $\mathrm{CC} / \mathrm{NCC}$ dichotomy are the main cause of the scatter in these profiles. The criteria for separating relaxed and un-relaxed clusters are based on three indicators: the virial ratio $\eta$, the centre-of-mass offset $\Delta_{r}$ and the fraction of mass in subhaloes $f_{\mathrm{s}}$. We adopt the same limitations as Cui et al. (2017) to select dynamically relaxed clusters: $0.85<\eta<1.15, \Delta_{r}<0.04$, and $f_{\mathrm{s}}<0.1$. While the CC and NCC clusters are separated by measuring the shape and level of the entropy profiles in the cluster central regions: the pseudo-entropy $\sigma$ and the central entropy $K_{0}$. The CC clusters are selected with $\sigma<0.55$ (Rasia et al. 2015) and $K_{0}<60 \mathrm{keV} \mathrm{cm}^{2}$ (Cavagnolo et al. 2009).

\section{A1 The effects of the cluster dynamical states on stellar density profile}

In Fig. A1, we show the stellar density profiles from relaxed (filled symbols and solid lines) and un-relaxed clusters (open symbols and dashed lines). We compare the two hydro-simulations in the left-hand panel and the three SAMs in the right-hand panel for a better visualization. The relaxed clusters tend to have a shallower profile than the un-relaxed clusters, especially at outer radii. There is almost no difference in the cluster centre region for the two hydrosimulations, but the stellar density is slightly higher for the relaxed clusters in the SAMs. However, the scatter is basically at the same level for the relaxed and un-relaxed clusters: $\sim 0.5-0.1$ dex for the hydro-simulations and for the SAMs. The difference between the 


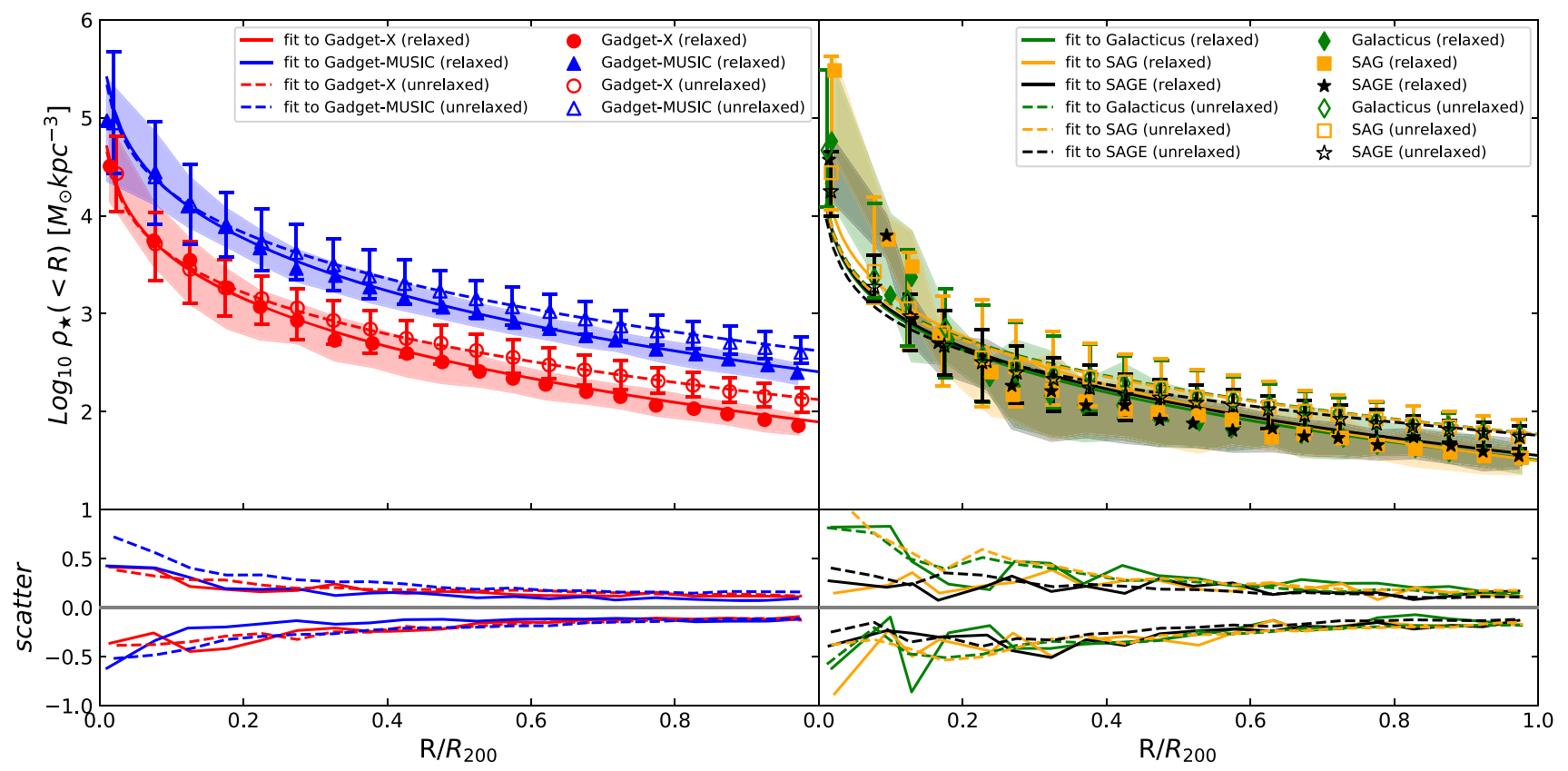

Figure A1. Cumulative stellar density profile for relaxed clusters and un-relaxed clusters. Left-hand panel shows the results from the two hydro-simulations, while right-hand panel is for the three SAMs. Shaded areas and error bars show the 16th and 84th percentiles for relaxed and un-relaxed clusters, respectively, while lower panels highlight their scatters. Solid and dashed lines, respectively, indicate the fitting for relaxed and un-relaxed clusters, using the formula from Łokas \& Mamon (2001). The fitting results are shown in Table A1.

relaxed and un-relaxed cluster profiles indicates that the dynamical state has an impact on the stellar density profiles.

\section{A2 The effects on gas density profile}

Gas density profiles are separated according to the cluster dynamical state in Fig. A2 and according to the CC/NCC dichotomy in Fig. A3. Since there is very little scatter in the gas density profiles at outer radii, we do not expect to see much difference at these radii. There is a clear separation in the cluster centre region - un-relaxed and NCC clusters tend to have a lower density profile compared to relaxed and $\mathrm{CC}$ clusters. Thus, only the inner gas density profile is influenced by the cluster dynamical state; the different trends of CC and NCC objects are in agreement with Ghirardini et al. (2019).

\section{A3 The effects on gas temperature profile}

As with the gas density profiles, we also find that the dependence of the cluster dynamical state and CC/NCC dichotomy on gas temperature is much stronger in the cluster centre than at outer radii.

In both simulations, the dynamical relaxed clusters have a slightly higher temperature than these un-relaxed clusters, which tends to be more obviously in the centre than the outer radii. The scatter seems a little lower for the dynamical relaxed clusters than these un-relaxed clusters.
It is not surprising to see that the NCC clusters tend to have a higher temperature in the cluster centre than the $\mathrm{CC}$ clusters. However, unlike the gas density profile, the un-relaxed clusters tend to have a lower temperature. This could be understood as the gas in the un-relaxed clusters still being in the process of shock heating. As shown in Fig. A5, the CC clusters in GADGET-X are in perfect agreement with Ghirardini et al. (2019). The NCC clusters in GADGET-X seem to be lower than the result from Ghirardini et al. (2019) and have an opposite trend at outer radii, i.e. a lightly lower temperature rather than a higher temperature than the $\mathrm{CC}$ clusters which is presented in the observation result. Again, although GADGET-MUSIC give a similar result, it is systematically lower than the observations at outer radii which is caused by the normalization.

\section{A4 The effects on gas metallicity profile}

As we expected, there is also not much difference between the metallicity profiles at the cluster outer radii between relaxed and un-relaxed clusters or CC and NCC clusters. While, in the cluster centre region, the differences are also very weak compared to the gas density or temperature profiles. In agreement with Lovisari \& Reiprich (2019), relaxed clusters tend to have a higher metallicity in the centre for both GADGET-X and GADGET-MUSIC. However, given the large error bar, we would like to conclude that gas metallicity is likely to be less affected by the cluster dynamical state or CC/NCC dichotomy. 
Table A1. The NFW fitting function and parameters for the cumulative density profiles in Fig. A1. The fitting process is the same as for Fig. 2. We note here that only $c$ is the free parameter in the fitting function.

\begin{tabular}{lcccccc}
\hline $\begin{array}{l}\text { Fitting parameters } \\
\begin{array}{l}\text { Cluster dynamical state } \\
\text { Model }\end{array}\end{array}$ & $c$ & $\begin{array}{c}10^{-3} R_{200}(\mathrm{kpc}) \\
\text { Relaxed }\end{array}$ & $10^{-12} M_{200}\left(\mathrm{M}_{\odot}\right)$ & $c$ & $10^{-3} R_{200}(\mathrm{kpc})$ \\
Un-relaxed & $10^{-12} M_{200}\left(\mathrm{M}_{\odot}\right)$ \\
\hline GADGET-X & 2.880 & 2.204 & 3.524 & 1.478 & 2.261 & 6.437 \\
GADGET-MUSIC & 4.285 & 2.221 & 11.723 & 4.991 & 2.260 & 20.209 \\
GALACTICUS & 1.687 & 2.196 & 1.431 & 2.589 & 2.253 & 2.820 \\
SAG & 3.017 & 2.196 & 1.394 & 0.832 & 2.253 & 2.795 \\
SAGE & 1.571 & 2.196 & 1.585 & 0.453 & 2.253 & 2.747 \\
Fitting function & \multicolumn{2}{c}{$\rho(s)=M_{200} g(c)\left(\ln (1+c s)-\frac{c s}{1+c s}\right) /\left(\frac{4}{3} \pi s^{3} r_{200}^{3}\right)$} \\
\end{tabular}

Table A2. The double $\beta$-model fitting function (based on Mohr et al. 1999) and the fitting parameters for Fig. A2.

\begin{tabular}{|c|c|c|c|c|c|c|}
\hline \multicolumn{2}{|c|}{ Fitting parameters } & \multirow[t]{2}{*}{$\rho_{1}\left(\mathrm{M}_{\odot} \mathrm{kpc}^{-3}\right)$} & \multirow[t]{2}{*}{$R_{1}\left(R_{500}\right)$} & \multirow[t]{2}{*}{$\rho_{2}\left(\mathrm{M}_{\odot} \mathrm{kpc}^{-3}\right)$} & \multirow[t]{2}{*}{$R_{2}\left(R_{500}\right)$} & \multirow[t]{2}{*}{$\beta$} \\
\hline Model & Dynamical state & & & & & \\
\hline \multirow[t]{2}{*}{ GADGET-X } & Relaxed & $3.679 \times 10^{5}$ & 0.034 & $2.291 \times 10^{5}$ & 0.143 & 0.757 \\
\hline & Un-relaxed & $2.038 \times 10^{5}$ & 0.059 & $7.434 \times 10^{4}$ & 0.239 & 0.742 \\
\hline \multirow[t]{2}{*}{ GADGET-MUSIC } & Relaxed & $3.606 \times 10^{5}$ & 0.071 & $7.259 \times 10^{4}$ & 0.220 & 0.812 \\
\hline & Un-relaxed & $2.539 \times 10^{5}$ & 0.046 & $7.348 \times 10^{4}$ & 0.225 & 0.748 \\
\hline Fitting function & \multicolumn{6}{|c|}{$\rho(R)=\sum_{i=1}^{2} \rho_{i}\left[1+\left(\frac{R}{R_{i}}\right)^{2}\right]^{-3 \beta / 2}$} \\
\hline
\end{tabular}

Table A3. Similar to Table A2 with fitting parameters for Fig. A3.

\begin{tabular}{lcccccc}
\hline \multicolumn{2}{c}{ Fitting parameters } & $\rho_{1}\left(\mathrm{M}_{\odot} \mathrm{kpc}^{-3}\right)$ & $R_{1}\left(R_{500}\right)$ & $\rho_{2}\left(\mathrm{M}_{\odot} \mathrm{kpc}^{-3}\right)$ & $R_{2}\left(R_{500}\right)$ & $\beta$ \\
Model & Core type & & & & & \\
\hline \multirow{2}{*}{ GADGET-X } & $\mathrm{CC}$ & $6.131 \times 10^{4}$ & 0.276 & $8.181 \times 10^{5}$ & 0.043 & 0.796 \\
& $\mathrm{NCC}$ & $9.330 \times 10^{4}$ & 0.222 & $1.072 \times 10^{5}$ & 0.066 & 0.754 \\
\multirow{2}{*}{ GADGET-MUSIC } & $\mathrm{CC}$ & $5.056 \times 10^{5}$ & 0.060 & $4.369 \times 10^{4}$ & 0.288 & 0.813 \\
& $\mathrm{NCC}$ & $8.848 \times 10^{4}$ & 0.207 & $2.897 \times 10^{5}$ & 0.040 & 0.751 \\
Fitting function & & & & \\
\hline
\end{tabular}

Table A4. The fitting function and parameters for Fig. A4. The fitting formula is from Ghirardini et al. (2019).

\begin{tabular}{|c|c|c|c|c|c|c|c|}
\hline \multicolumn{2}{|c|}{ Fitting parameters } & \multirow[t]{2}{*}{$T_{0}\left(T_{500}\right)$} & \multirow[t]{2}{*}{$T_{\min }\left(T_{500}\right)$} & \multirow[t]{2}{*}{$r_{\text {cool }}\left(R_{500}\right)$} & \multirow[t]{2}{*}{$a_{\text {cool }}$} & \multirow[t]{2}{*}{$r_{t}\left(R_{500}\right)$} & \multirow[t]{2}{*}{$c$} \\
\hline Model & Dynamical state & & & & & & \\
\hline \multirow[t]{2}{*}{ GADGET-X } & Relaxed & 0.502 & 1.083 & 3.363 & 15.771 & 0.461 & 0.576 \\
\hline & Un-relaxed & 1.041 & 0.619 & 0.021 & 2.965 & 0.330 & 0.440 \\
\hline \multirow[t]{2}{*}{ GADGET-MUSIC } & Relaxed & 1.139 & 0.502 & 0.003 & 28.153 & 0.299 & 0.774 \\
\hline & Un-relaxed & 0.747 & 1.040 & 0.124 & 1.464 & 1.243 & 2.847 \\
\hline Fitting function & \multicolumn{7}{|c|}{$\frac{T(x)}{T_{500}}=T_{0} \frac{\frac{T_{\min }}{T_{0}}+\left(\frac{x}{r_{\text {cool }}}\right)^{a_{\text {cool }}}}{1+\left(\frac{x}{r_{\text {cool }}}\right)^{a_{\text {cool }}}} \frac{1}{\left(1+\left(\frac{x}{r_{t}}\right)^{2}\right)^{\frac{c}{2}}}$} \\
\hline
\end{tabular}


Table A5. The fitting function and parameters for Fig. A5. The fitting formula is from Ghirardini et al. (2019). The thick black solid and dashed lines are the CC and NCC clusters from Ghirardini et al. (2019), respectively.

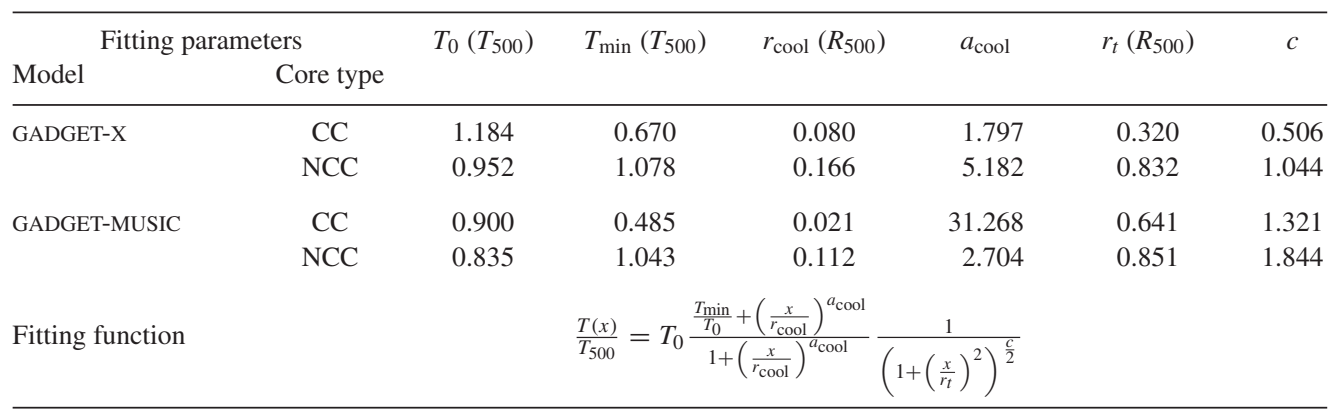

Table A6. The fitting function and parameters for Fig. A6.

\begin{tabular}{lcccc}
\hline Fitting parameters & \multicolumn{2}{c}{ GADGET-X } & \multicolumn{2}{c}{ GADGET-MUSIC } \\
\hline Dynamical state & Relaxed & Un-relaxed & Relaxed & Un-relaxed \\
$Z_{0}\left(\mathrm{Z}_{\odot}\right)$ & 0.060 & 0.146 & 0.200 & 0.092 \\
$R_{0}\left(R_{500}\right)$ & 2.010 & 0.591 & 0.146 & 0.448 \\
$b$ & 3.254 & 2.192 & 2.669 & 3.183 \\
& \multicolumn{3}{c}{$Z(R)=Z_{0} \mathrm{e}^{-b\left[\left(\frac{R}{R_{0}}\right)^{\frac{1}{4}}-1\right]}$} \\
Fitting function & \multicolumn{3}{c}{} \\
\hline
\end{tabular}

Table A7. The fitting function and parameters for Fig. A7.

\begin{tabular}{lcccc}
\hline Fitting parameters & \multicolumn{2}{c}{ GADGET-X } & \multicolumn{2}{c}{ GADGET-MUSIC } \\
\hline Core type & CC & NCC & CC & NCC \\
$Z_{0}\left(\mathrm{Z}_{\odot}\right)$ & 0.195 & 0.130 & 0.171 & 0.091 \\
$R_{0}\left(R_{500}\right)$ & 0.297 & 0.816 & 0.168 & 0.447 \\
$b$ & 2.170 & 2.192 & 2.581 & 3.253 \\
& & & \\
Fitting function & \multicolumn{2}{c}{$Z(R)=Z_{0} \mathrm{e}^{-b\left[\left(\frac{R}{R_{0}}\right)^{\frac{1}{4}}-1\right]}$} \\
\hline
\end{tabular}

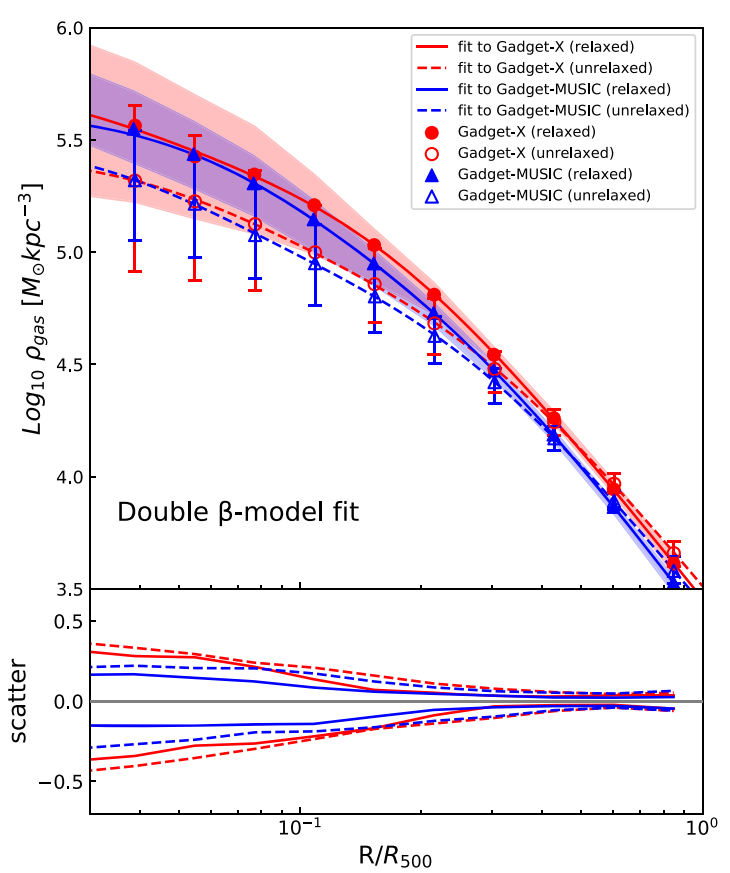

Figure A2. Radial gas density profile for relaxed clusters and un-relaxed clusters from the two hydro-simulations. Upper panel presents the results with shaded areas and error bars indicating the 16th and 84th percentiles for relaxed and un-relaxed clusters, respectively, while the lower panel highlights these scatters. Solid and dashed lines, respectively, indicate the fitting for relaxed and up-relaxed cluster with double $\beta$-model. The fitting results are shown in Table A2. 


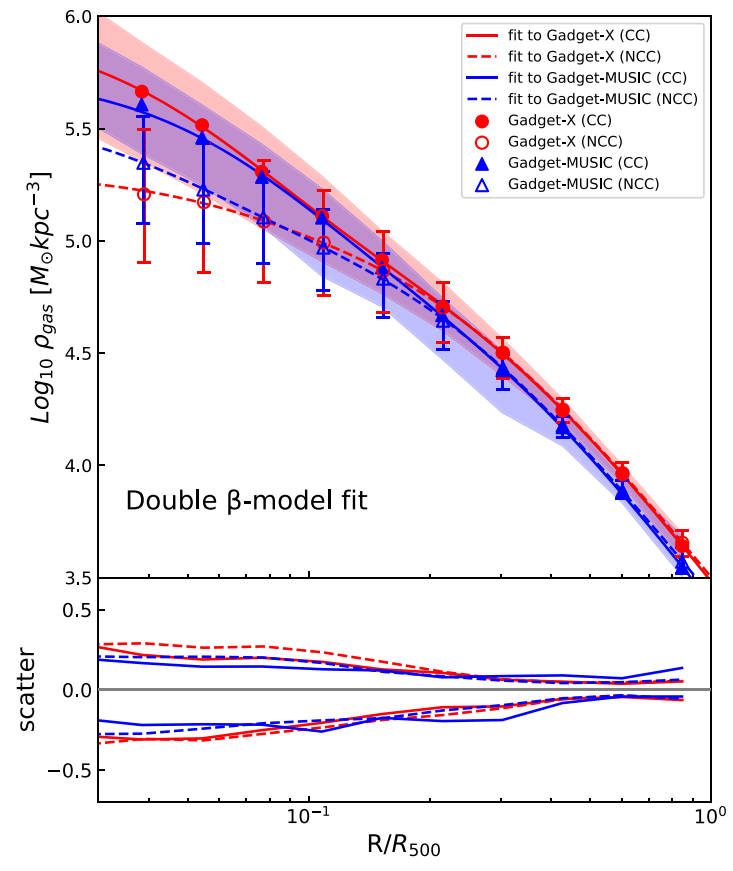

Figure A3. Similar to Fig. A2 with a separation of cool-core and non-coolcore clusters. The fitting results are shown in Table A3.

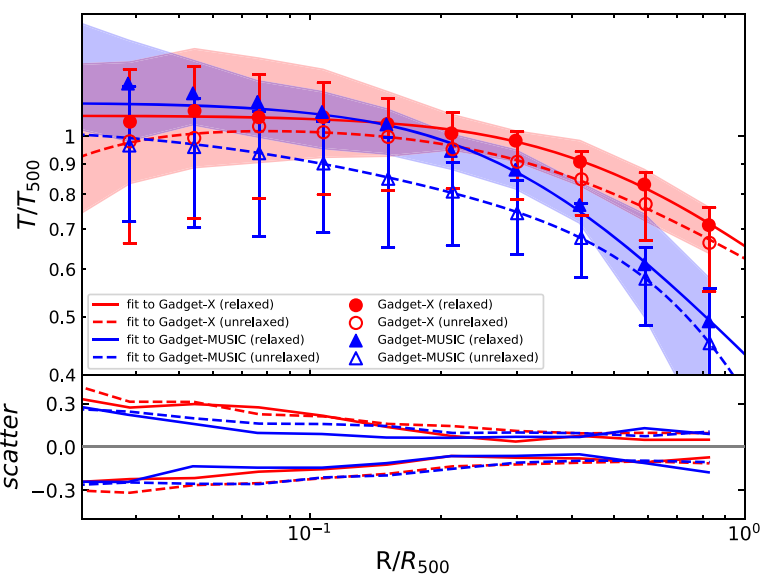

Figure A4. Similar to Fig. A2 but for the gas temperature profile of relaxed clusters and un-relaxed clusters. The fitting results shown by solid and dashed lines use the function from Ghirardini et al. (2019) and are listed in Table A4.

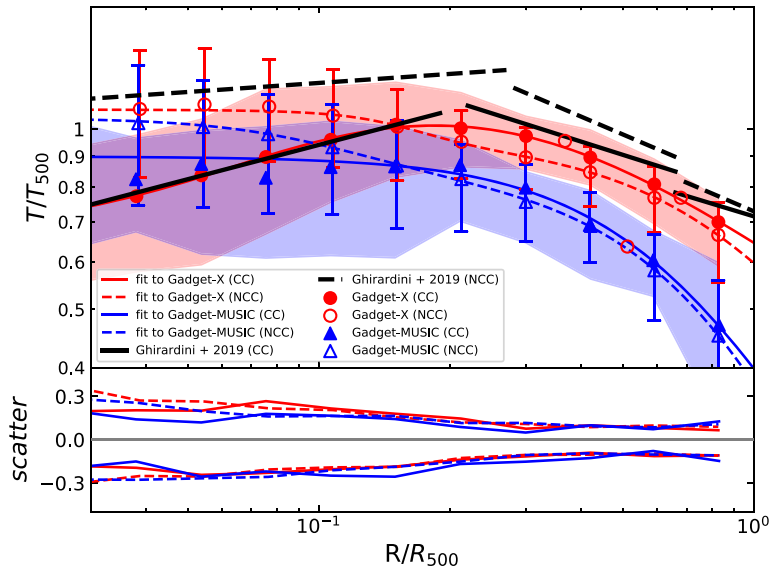

Figure A5. Similar to Fig. A2 but for the gas temperature profiles, separated according to cool-core clusters and non-cool-core clusters. The fitting results shown as solid and dashed lines use the equation from Ghirardini et al. (2019) and are listed in Table A5.

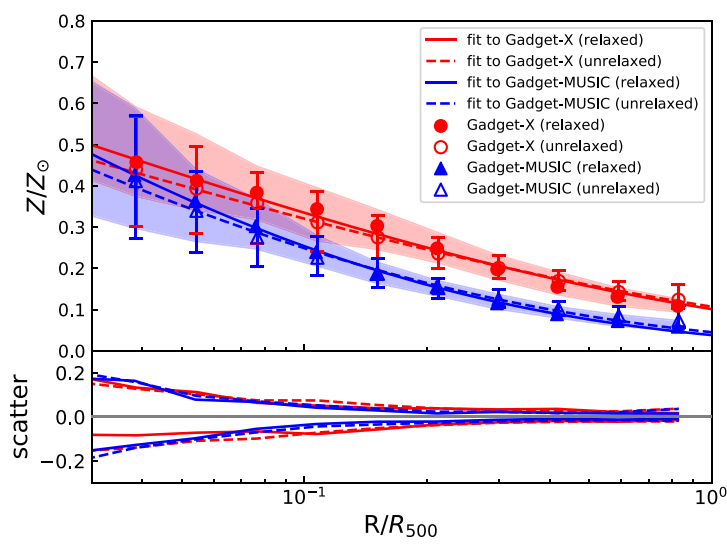

Figure A6. Similar to Fig. A2 but for the gas metallicity profile of relaxed clusters and un-relaxed clusters. The fitting results shown as solid and dashed lines use the Sérsic profile (Sérsic 1963) and are listed in Table A6.

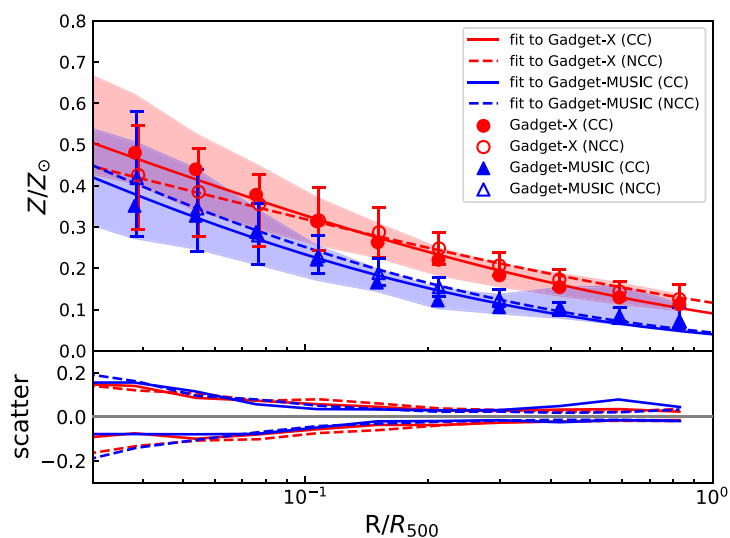

Figure A7. Similar to Fig. A2 but for the gas metallicity profile of $\mathrm{CC}$ and NCC clusters. The fitting results shown as solid and dashed lines use the Sérsic profile (Sérsic 1963) and are listed in Table A7. 


\section{APPENDIX B: THE $T_{500}$ DIFFERENCE}

In Fig. B1, we show the ratio of cluster temperature $T_{500}$ between two different definitions which are illustrated in Section 4.2.2. The $T_{500, G+19}$ temperature is based on cluster mass $M_{500}$ (see equation 8 for details), while $T_{500, \mathrm{sl}}$ is calculated by spectroscopic weighted temperature using gas particles within a sphere of radius $R_{500}$. It is clear that $T_{500, G+19}$ is generally higher ( $\sim 1.5$ times) than $T_{500, \mathrm{sl}}$ with GADGET-MUSIC tends to have a higher difference and a larger scatter than GADGET-X. We do not see a clear mass dependence. By separating the clusters into relaxed and un-relaxed, it is clear that these clusters with large difference are basically un-relaxed clusters, which tend to give much larger biased halo mass estimated from the hydrostatic equilibrium assumption.

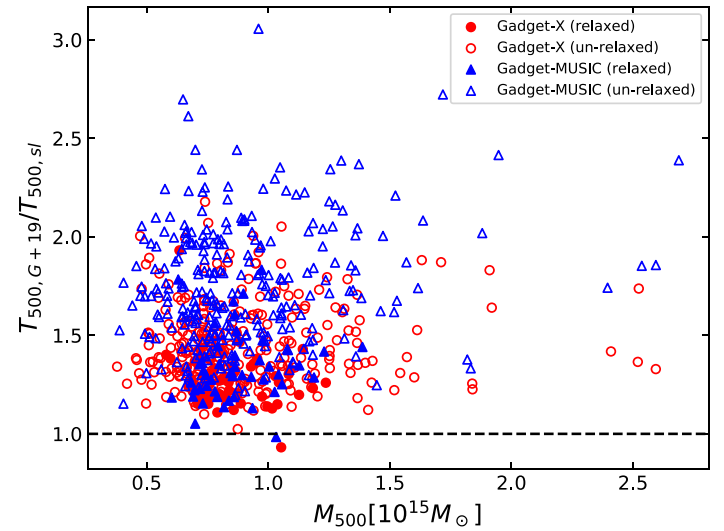

Figure B1. The ratio between $T_{500, G+19}$ and $T_{500, \mathrm{sl}}$ as a function of halo mass $M_{500}$. Each symbol represents a cluster from GADGET-X (red circle) and GADGET-MUSIC (blue triangle) with filled for relaxed clusters and opened for un-relaxed clusters.

This paper has been typeset from a TEX/LTEX file prepared by the author. 